\title{
Systematic Review with Meta-analysis: Association of Helicobacter pylori Infection with Esophageal Cancer
}

\author{
Huiqin Gao $\mathbb{D}^{1,2}$ Lunan Li, ${ }^{1,}$ Chenjing Zhang, ${ }^{1}$ Jiangfeng Tu, ${ }^{1}$ Xiaoge Geng, ${ }^{1}$ Jingya Wang, \\ Xiaolu Zhou, ${ }^{1}$ Jiyong Jing ${ }^{1},{ }^{3}$ and Wensheng Pan ${ }^{1}{ }^{1}$ \\ ${ }^{1}$ Department of Gastroenterology, Zhejiang Provincial People’s Hospital, People's Hospital of Hangzhou Medical College, Hangzhou, \\ Zhejiang, China \\ ${ }^{2}$ Bengbu Medical College, Bengbu, Anhui, China \\ ${ }^{3}$ Zhejiang Provincial People's Hospital, People's Hospital of Hangzhou Medical College, Hangzhou, Zhejiang, China
}

Correspondence should be addressed to Jiyong Jing; jiyong_jing@126.com and Wensheng Pan; wspan223@163.com

Received 9 July 2019; Revised 21 October 2019; Accepted 8 November 2019; Published 1 December 2019

Academic Editor: Eiji Sakai

Copyright (c) 2019 Huiqin Gao et al. This is an open access article distributed under the Creative Commons Attribution License, which permits unrestricted use, distribution, and reproduction in any medium, provided the original work is properly cited.

Background. Helicobacter pylori is an important carcinogenic factor in gastric cancer. Studies have shown that Helicobacter pylori infection is inversely associated with certain diseases such as esophageal cancer and whose infection appears to have a "protective effect." At present, the relationship between Helicobacter pylori infection and esophageal cancer remains controversial. This study was designed to investigate the relationship between Helicobacter pylori infection and the risk of esophageal cancer in different regions and ethnicities. Methods. Systematic search of the articles on the relationship between Helicobacter pylori infection and esophageal cancer from the database with the duration time up to December 2018. This systematic review was performed under the MOOSE guidelines. Results. This meta-analysis included 35 studies with 345,886 patients enrolled. There was no significant correlation between Helicobacter pylori infection and esophageal squamous cell carcinoma in the general population (OR: 0.84; 95\% CI: 0.64-1.09/OR: 0.74; 95\% CI: 0.54-0.97). However, a significant correlation was found in the Middle East (OR: 0.34; 95\% CI: $0.22-0.52 / 95 \%$ CI: $0.26-0.44)$. There was no significant difference in the prevalence of Helicobacter pylori between the case group and the control group in esophageal adenocarcinoma ( $8.87 \%$ vs. $9.67 \%)$. The pooled OR was 0.55 (95\% CI: $0.43-0.70)$ or 0.23 (95\% CI: $0.15-0.36$ ). When grouped by match or not, the pooled OR of the nonmatching group and the matching group was $0.48 / 0.21$ (95\% CI: $0.36-0.65 / 95 \%$ CI: $0.13-0.36)$ and $0.73 / 0.71$ (95\% CI: $0.57-0.92 / 95 \%$ CI: $0.60-0.84)$, respectively. Conclusion. In the general populations, no significant association was found between Helicobacter pylori infection and the risk of esophageal squamous cell carcinoma. However, lower risk was found in the Middle East. Helicobacter pylori infection may reduce the risk of esophageal adenocarcinoma, but such "protection effect" may be overestimated.

\section{Introduction}

Esophageal cancer ranks the eighth in the world's cancer incidence and the sixth in the global cancer death cause [1]. There are two major histological subtypes of the esophagus: esophageal squamous cell carcinoma (ESCC) and esophageal adenocarcinoma (EAC). ESCC and EAC have different geographic and demographic models. ESCC has a high incidence in many developing countries. The most important risk factors in Western countries are smoking and habitual consumption of alcohol. In developed countries such as North America, Australia, and Europe, esophageal adenocar- cinoma has become the main subtype of esophageal cancer; its major risk factors include chronic gastroesophageal reflux disease, obesity, and smoking [2-4].

Helicobacter pylori is a common bacterium in the upper digestive tract, which infects about half of the world [5]. Marshall and Warren first reported the cultivation of Helicobacter pylori from human gastric mucosa in 1983 [6]. The International Agency for Research on Cancer and the World Health Organization believed that Helicobacter pylori is a carcinogen of gastric cancer [7]. However, some studies have shown that Helicobacter pylori infection is negatively correlated with some diseases [8-11]. Helicobacter pylori 
infection appeared to have a "protective effect." Since the 20th century, the prevalence of Helicobacter pylori has declined in Western countries; the incidence of esophageal cancer has subsequently increased. Although the previous meta-analysis has systematically illustrated on the relationship between them, there has been controversy [12-15]. At present, the relationship between Helicobacter pylori and esophageal squamous cell carcinoma has not been clearly explained; the evidence of its protective or harmful effects on esophageal adenocarcinoma is still contradictory. In recent years, articles on the relationship between Helicobacter pylori and esophageal cancer have been published in succession; new data can be used to further analyze the relationship between Helicobacter pylori and esophageal cancer. Moreover, whether there are different relations between different regions and ethnicities has not been specifically explained. Therefore, we did a meta-analysis to explore the relationship between Helicobacter pylori infection and the risk of esophageal cancer in different regions and ethnicities.

\section{Methods}

The data of this meta-analysis were collected based on the Meta-analysis of Observational Studies in Epidemiology (MOOSE) statements [16] (Table S3).

2.1. Data Source and Search Strategy. All articles and abstracts published up to December 2018 were systematically searched in Embase and PubMed using MeSH terms and free words. Some of the database search MeSH terms are the following: "Esophageal Neoplasms", "Esophageal squamous cell carcinoma", "Adenocarcinoma of Esophagus", and "Helicobacter pylori". Comprehensive search terms are listed in the appendix. Besides, manual searches were conducted to ensure that all articles were related to our subject. Other sources are from related articles mentioned in the previous related metaanalysis [17-20]. There are no predetermined study design types, language restrictions, or publication years.

2.2. Study Selection. The two authors (HQ Gao and LN Li) independently selected articles to be included and conducted critical assessments. Discrepancies were resolved by reaching a consensus with the senior author (JY Jing). Eligible studies were selected according to the following inclusion criteria: (1) they are studies on the relationship between Helicobacter pylori infection and esophageal cancer, (2) the incidence of esophageal cancer in the control and case groups can be extracted when exposed to or not exposed to Helicobacter pylori, (3) the odds ratio (OR) (case-control study) or relative risk (RR) (cohort study) and 95\% confidence interval (95\% CI) can be directly or indirectly calculated, and (4) the full text can be obtained. The exclusion criteria are (1) unobtainable full text, (2) the repeated study of the same population sample, and (3) the secondary data analysis literature such as review or meta-analysis.

2.3. Data Extraction. Data extraction was independently completed by the two researchers (HQ Gao and LN Li). First of all, after the thorough review of the title and abstract, obtain the full-text literature which meets the inclusion criteria. Two sides discuss for the controversial literature and seek the third senior researcher (JY Jing) to make a final decision if controversies were meet. The following data were extracted from each article: first author's name, year of publication, study country, ethnicity, study type, study subject, Helicobacter pylori test method, and number of control and case groups, as well as related measurement indicators (OR or RR and $95 \% \mathrm{CI}$ ).

2.4. Quality Assessment. Two authors (HQ Gao and LN Li) independently evaluated the quality of the articles. Discrepancies were resolved by reaching a consensus with the senior author (JY Jing). The quality of the literature was evaluated using the Newcastle-Ottawa Scale (NOS) [21]. The scale was evaluated from study population selection (4 points), intergroup comparability ( 2 points), and exposure or outcome evaluation ( 3 points). The total score is 9 points, with higher scores indicating better methodological quality.

2.5. Risk of Bias Assessment. The two authors (HQ Gao and $\mathrm{LN} \mathrm{Li}$ ) performed the risk assessment of the included studies. Any disputes or inconsistencies were discussed in the group to achieve a consistent result. The risk of bias was evaluated by the ROBINS-I (Risk Of Bias In Nonrandomized Studies-of Interventions) tool [22]. Based on the risk of bias of seven different domains, the overall bias risk for each outcome and study was estimated. It is divided into three parts: preintervention (bias due to confounding, bias in selection of participants into the study), at intervention (bias in classification of interventions), and postintervention (bias due to deviations from intended interventions, bias due to missing data, bias in measurement of outcomes, and bias in selection of the reported result).

2.6. Statistical Analysis. The relationship between Helicobacter pylori and esophageal cancer was summarized by the binary method. OR and 95\% CI were calculated for each study. To investigate the sources of heterogeneity, we carried out the following tests: heterogeneity tests, subgroup analysis, metaregression analysis, and sensitivity analysis. The heterogeneity of each study was statistically analyzed by $Q$ test and $I^{2}$ test. When $I^{2}$ was $0-40 \%$, it means littler no heterogeneity, 30-60\% means moderate heterogeneity, $50-90 \%$ indicates substantial heterogeneity, and $75-100 \%$ indicates considerable heterogeneity [23]. If there is moderate heterogeneity or above, we use the HartungKnapp-Sidik-Jonkman (HKSJ) method for further statistics [24]. The random effects model is used to pool the analysis [25], and subgroup analysis is carried out according to the factors that may cause heterogeneity (ethnicity, study area, study type, study object, Helicobacter pylori detection method, and whether the study population of control group and case group is matched by gender, age, and race). Subsequently, restricted maximum likelihood-based random effects metaregression analyses were carried out to evaluate potential heterogeneous factors. Univariate metaregression analysis was conducted first, after which the variables that were significant at the 0.1 level were entered into the multivariable model. Sensitivity analysis verifies the stability of 
the results by eliminating each study one by one. Assessment of potential publication bias was made using Harbord weighted linear regression and funnel plots. Data collation and analysis were performed using Stata13.1 software and RStudio software.

\section{Results}

3.1. Characteristics of Studies. According to the inclusion and exclusion criteria, a total of 35 studies were included in our study [17-20, 26-56]. A flow diagram for our systematic review is shown in Figure 1. Table 1 summarized the baseline characteristics of the included studies. Twelve studies from North America [17-20, 28, 40,46, 48, 50, 54-56], ten studies from Europe [26, 32, 33, 36, 39, 42, 43, 49, 52, 53], eight studies from East Asia [27, 31, 37, 38, 44, 45, 47, 51], four studies from the Middle East [29, 30, 34, 41], and one study from Oceania [35]. Regarding the study type, thirty-three of them are case-control studies $[17-20,27-30,32-56]$ and two of them are cohort studies [26, 31]. Table 2(a) summarized the data of Helicobacter pylori infection in esophageal squamous cell carcinoma, with a total of 37,114 patients, including 2,063 patients in the case group and 35,051 patients in the control group. Table 2(b) contains the data of esophageal adenocarcinoma, with a total of 308,772 patients, including 7,687 patients in the case group and 301,085 patients in the control group. Furthermore, 14 studies involved esophageal adenocarcinoma $[18,19,28,39-42,46,48,50,53-56], 15$ studies involved esophageal squamous cell carcinoma [17, 27, 29-34, 36-38, 44, 45, 47, 51], and 6 studies involved both types $[20,26,35,43,49,52]$. According to the NewcastleOttawa Scale, sixteen studies were ranked as very good [17, $19,20,26,29,32,33,35,39,40,43,44,48-50,56]$, seventeen as good [18, 28, 31, 34, 36-38, 41, 42, 45-47, 51-55], and two as satisfactory $[27,30]$ (Table S1).

3.2. Risk of Bias within Studies. According to the ROBINS-I tool, four studies had a serious risk of bias $[19,34,37,46]$, 26 studies had a moderate risk of bias $[17,18,20,26,27$, $29-31,33,35,38-43,45,47-51,53-56]$, and five of the other studies had a low risk of bias $[28,32,36,44,52]$ (Table S2).

3.3. Helicobacter pylori Infection and the Risk of Esophageal Squamous Cell Carcinoma. Twenty-one studies with 37,114 patients reported the relationship between Helicobacter pylori and esophageal squamous cell carcinoma $[17,20$, $26,27,29-38,43-45,47,49,51,52]$. The prevalence of Helicobacter pylori infection in the case group was higher than that in the control group $(1,059 / 2,063(51.33 \%)$ vs. $13,688 / 35,051(39.05 \%))$. No statistical significance was showed according to the DerSimonian-Laird method (OR: 0.84, 95\% CI: 0.64-1.09). There was no significant association between Helicobacter pylori infection and the risk of esophageal squamous cell carcinoma according to ethnicity, study type, and matching, which had no statistically significant differences between the groups. However, in the Middle East, significantly lower risk of esophageal squamous cell carcinoma was observed after grouping by the study area (OR: $0.34,95 \%$ CI: 0.22-0.52), while that in North America showed the opposite-higher risk (OR: 1.83, 95\% CI: 1.17-2.87). Besides, no significant correlation was found in East Asia, Europe, and Australia. Above all, there were significant statistical differences among regions $(P<0.001)$. When analyzing population-based studies, the correlation coefficient between Helicobacter pylori infection and the risk of ESCC was 0.93 (95\% CI: 0.68-1.28). Based on clinical studies, with a pooled OR of $0.66(95 \%$ CI: $0.40-1.07)$. There were significant differences between the groups $(P=0.005)$. After grouping based on the detection method of Helicobacter pylori, the pooled OR of the detection group with various methods was 1.32 (95\% CI: $0.87-2.01)$. There were significant differences between the groups $(P=0.003)$. According to the HKSJ method, Helicobacter pylori did not increase the risk of esophageal squamous cell carcinoma in North America (OR: 1.79, 95\% CI: 0.25-12.9). There was no statistically significant difference between the remaining comparative according to the DL methods and the HKSJ method. (The specific results are shown in Table 3(a) and Figures 2(a) and 3(a).)

In the study of esophageal squamous cell carcinoma, the $Q$ statistic was significant $(P<0.001)$ and the $I^{2}$ statistic had a higher heterogeneity $\left(I^{2}=78.5 \%\right)$. Hence, we performed a subgroup analysis to further explore the potential source of heterogeneity. The heterogeneity did not decrease significantly when grouped according to ethnicity, study object, and matching, but it decreased significantly when grouped according to the study area, study type, and Helicobacter pylori detection method. The results of the sensitivity analysis showed that after excluding one study, the pooling effect of the remaining studies was basically the same as the original total pooling effect. This confirmed that our results were stable.

The results of the funnel plot showed no significant asymmetry (Figure $4(\mathrm{a})$ ), which suggested that the results were less likely to be affected by publication bias. Harbord's test showed that the $P$ values were 0.920 , which indicated that there was no significant publication bias in the whole study.

3.4. Helicobacter pylori Infection and the Risk of Esophageal Adenocarcinoma. A total of 308,772 patients in 20 studies of esophageal adenocarcinoma were enrolled in our study $[18-20,26,28,35,39-43,46,48-50,52-56]$. The prevalence of Helicobacter pylori infection in the case group was not significantly different from that in the control group $(682 / 7,687$ (8.87\%) vs. $29,109 / 301,085$ (9.67\%)). According to the DL method, the quantitative meta-analysis showed that the pooled ORs were 0.55 (95\% CI: 0.43-0.70). When grouped according to matching, the OR of the nonmatching group and matching group was 0.48 (95\% CI: $0.36-0.65)$ and 0.73 (95\% CI: 0.57-0.92), respectively. There were significant differences between the groups $(P<0.001)$. After grouping based on the detection method of Helicobacter pylori, the OR of the detection group with various methods was 0.81 (95\% CI: 0.32-2.07). There were significant differences between the groups $(P<0.001)$. Besides, the risk of Helicobacter pylori infection and esophageal adenocarcinoma was also negatively correlated after grouping based on ethnicity and study object; there were significant differences between 


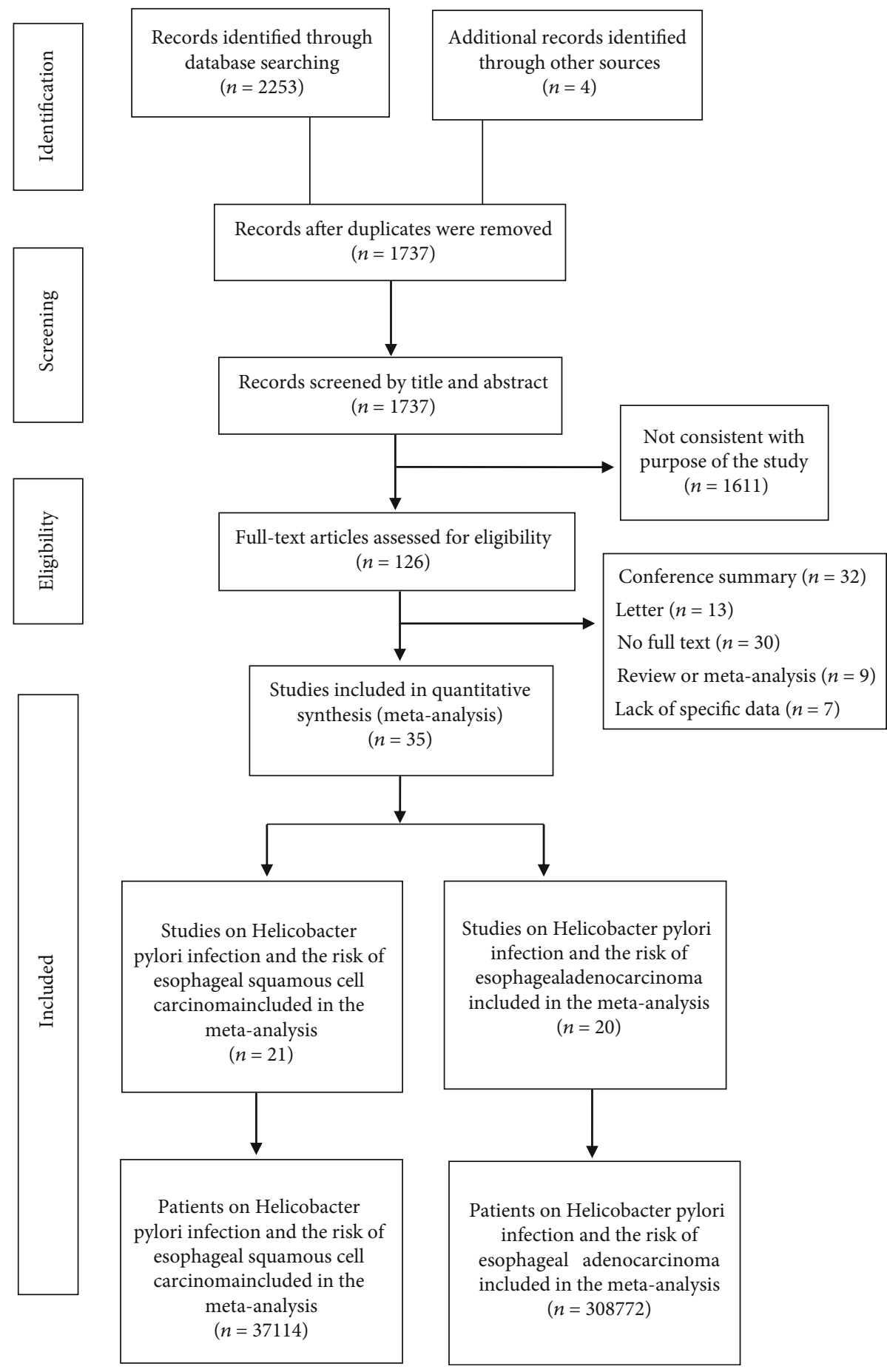

FIGURE 1: Flowchart showing selection of publications for review.

the groups $(P<0.001)$. According to the HKSJ method, the pooled OR of the nonmatching group was 0.21 (95\% CI: $0.13-0.36)$, while the OR of the matching group was 0.71 (95\% CI: 0.60-0.84). (The specific results are shown in Table 3(b) and Figures 2(b) and 3(b).)

In esophageal adenocarcinoma, the $Q$ statistic was significant $(P<0.001)$ and the $I^{2}$ statistic had a higher heterogeneity in the study results $\left(I^{2}=73.4 \%\right)$. The heterogeneity did not decrease significantly when grouped according to the study area and study type, but it decreased significantly when grouped according to ethnicity, study object, matching, and detection method of Helicobacter pylori. Besides, we did not find substantial changes in the corresponding pooled ORs by sensitivity analysis. This confirmed that our results were stable.

The results of the funnel plot showed slightly asymmetric signs (Figure 4(b)), which suggested that there may exist potential publication bias. But when we performed the trim 
TABLE 1: Characteristics of literatures included in the meta-analysis.

\begin{tabular}{|c|c|c|c|c|c|c|c|c|}
\hline First author & Study country & Ethnicity & Study type & Study object & Age* & $\operatorname{Sex}^{\Im}(\%$ male $)$ & Matched** & Follow-up \\
\hline Talley et al. & America & Caucasians & Case-control & Clin (S) & 63 & 49 & Yes & - \\
\hline Chow et al. & America & Caucasians & Case-control & Pop (A) & - & - & Yes & - \\
\hline Grimley et al. & America & Caucasians & Case-control & Clin (A) & 70 & $70-78$ & No & - \\
\hline Öberg et al. & America & Caucasians & Case-control & Clin (A) & - & - & No & - \\
\hline Peek et al. & America & Caucasians & Case-control & Clin (A) & 61 & 97 & No & - \\
\hline Vieth et al. & Germany & Caucasians & Case-control & Clin (A) & - & - & - & - \\
\hline Weston et al. & America & Caucasians & Case-control & Clin (A) & 60.6 & 100 & No & - \\
\hline Henrik et al. & Sweden & Caucasians & Case-control & Pop (S, A) & 49.6 & - & No & - \\
\hline Wu et al. & America & Caucasians & Case-control & Pop (A) & - & - & Yes & - \\
\hline El-Omar et al. & America & Caucasians & Case-control & Pop (S, A) & $\begin{array}{l}\text { S: } 66 \\
\text { A: } 65\end{array}$ & $\begin{array}{l}\text { S: } 89 \\
\text { A: } 86\end{array}$ & $\begin{array}{l}\text { S: No } \\
\text { A: Yes }\end{array}$ & - \\
\hline Wang et al. & China & Mongolians & Case-control & Pop (S) & - & - & No & - \\
\hline Ye et al. & Sweden & Caucasians & Case-control & Pop (S, A) & $\begin{array}{l}\text { S: } 64 \\
\text { A: } 69\end{array}$ & $\begin{array}{l}\text { S: } 69 \\
\text { A: } 91\end{array}$ & $\begin{array}{l}\text { No } \\
\text { No }\end{array}$ & - \\
\hline De Martel et al. & America & Caucasians & Case-control & Pop (A) & 47.9 & 80.4 & Yes & - \\
\hline Wu et al. & China & Mongolians & Case-control & Pop (S) & - & 91.3 & No & - \\
\hline Anandasabapathy et al. & America & Caucasians & Case-control & Clin $(\mathrm{A})$ & 59.23 & 94.3 & No & - \\
\hline Kamangar et al. & China & Mongolians & Case-control & Pop (S) & 54.5 & 46.3 & No & - \\
\hline Iijima et al. & Japan & Mongolians & Case-control & Clin (S) & 68.6 & 90.4 & No & - \\
\hline Simán et al. & Sweden & Caucasians & Case-control & Pop (S, A) & $\begin{array}{l}\text { S: } 50.6 \\
\text { A: } 49.3\end{array}$ & $\begin{array}{l}\text { S: } 81.1 \\
\text { A: } 91.7\end{array}$ & $\begin{array}{l}\text { No } \\
\text { No }\end{array}$ & - \\
\hline Anderson et al. & Ireland & Caucasians & Case-control & Pop (A) & 64.2 & 84 & No & - \\
\hline Früh et al. & Canada & Caucasians & Case-control & Clin (A) & 64 & 88 & Yes & - \\
\hline Löfdahl et al. & Sweden & Caucasians & Case-control & Pop (A) & - & - & No & - \\
\hline Derakhshan et al. & Iran & Caucasians & Case-control & Clin (A) & 63.9 & 63.2 & No & - \\
\hline Wu et al. & China & Mongolians & Case-control & Pop (S) & 58.3 & 95 & - & - \\
\hline Hu et al. & China & Mongolians & Case-control & Clin (S) & - & 97 & Yes & - \\
\hline Whiteman et al. & Australia & Australian & Case-control & Pop (S, A) & - & $\begin{array}{l}\text { S: } 58 \\
\text { A: } 92\end{array}$ & $\begin{array}{l}\text { No } \\
\text { No }\end{array}$ & - \\
\hline Cook et al. & Finland & Caucasians & Case-control & Pop (S) & 57.7 & - & No & - \\
\hline Venerito et al. & Germany & Caucasians & Case-control & Clin (S) & 64.9 & 69.3 & No & - \\
\hline Khoshbaten et al. & Iran & Caucasians & Case-control & Clin (S) & 63.9 & 64 & No & - \\
\hline Murphy et al. & Finland & Caucasians & Case-control & Pop (S) & 57.9 & - & No & - \\
\hline Xue et al. & China & Mongolians & Cohort & Pop (S) & 45.29 & 36.91 & - & $15 \mathrm{y}$ \\
\hline Obayo et al. & Uganda & Negroes & Case-control & Clin (S) & - & - & - & - \\
\hline Poyrazoglu et al. & Iran & Caucasians & Case-control & Clin (S) & - & - & No & - \\
\hline Sonnenberg et al. & America & Mixed race & Case-control & Clin (A) & 66.8 & 79.3 & No & - \\
\hline Tseng et al. & China & Mongolians & Case-control & Pop (S) & - & - & - & - \\
\hline Vohlonen et al. & Finland & Caucasians & Cohort & Pop (S, A) & - & 100 & No & $15 y$ \\
\hline
\end{tabular}

Note: *average or median age in case group; ${ }^{\S}$ the proportion of males in the case group; ${ }^{* *}$ does the case group match the age, sex, or race of the control group? Clin: clinical-based; Pop: population-based; S: esophageal squamous cell carcinoma; A: esophageal adenocarcinoma.

and fill method to identify and correct the asymmetry of the funnel plot caused by the potential publication bias, there was no possibility to perform the fill statistics, indicating that no publication bias was detected. Harbord's test showed that the $P$ value was 0.222 , which indicated that there was no significant publication bias in the whole study.

3.5. Metaregression. In order to further study the effects of these characteristics on estimating the relationship between esophageal cancer and Helicobacter pylori, we conducted the metaregression analysis. Year, ethnicity, study area, study type, study object, matching, and Helicobacter pylori detection method were entered as explanatory covariates. First of all, univariate metaregression analysis was performed. Regarding esophageal squamous cell carcinoma, in univariate metaregression analysis, year $(P=0.135)$, ethnicity (Caucasians: $P=0.394$, Mongolians: $P=0.341$, and Australian: $P=0.279$ ), study area (North America: 
TABLE 2

(a) The data of Helicobacter pylori infection in esophageal squamous cell carcinoma

\begin{tabular}{|c|c|c|c|c|c|c|}
\hline \multirow{3}{*}{ First author } & \multirow{3}{*}{ Year } & \multicolumn{4}{|c|}{$\begin{array}{c}\text { Esophageal squamous cell } \\
\text { carcinoma }\end{array}$} & \multirow{3}{*}{$\begin{array}{c}\text { Hp } \\
\text { Test method }\end{array}$} \\
\hline & & \multicolumn{2}{|c|}{ Case } & \multicolumn{2}{|c|}{ Control } & \\
\hline & & $\mathrm{Hp}+$ & Hp- & $\mathrm{Hp}+$ & Hp- & \\
\hline Talley et al. & 1991 & 20 & 21 & 96 & 156 & $S$ \\
\hline Henrik et al. & 2001 & 10 & 19 & 67 & 82 & S \\
\hline El-Omar et al. & 2003 & 31 & 22 & 84 & 126 & S \\
\hline Wang et al. & 2003 & 33 & 30 & 145 & 165 & S \\
\hline Ye et al. & 2004 & 32 & 53 & 198 & 301 & S \\
\hline Wu et al. & 2005 & 28 & 99 & 74 & 97 & S \\
\hline Kamangar et al. & 2007 & 254 & 81 & 662 & 330 & $S$ \\
\hline Iijima et al. & 2007 & 60 & 13 & 56 & 17 & $\mathrm{~S}, \mathrm{H}, \mathrm{U}$ \\
\hline Simán et al. & 2007 & 15 & 22 & 68 & 61 & S \\
\hline Wu et al. & 2009 & 112 & 205 & 563 & 540 & $S$ \\
\hline Hu et al. & 2009 & 66 & 114 & 102 & 92 & S \\
\hline Whiteman et al. & 2010 & 54 & 154 & 302 & 1,014 & S \\
\hline Cook et al. & 2010 & 64 & 14 & 71 & 20 & S \\
\hline Venerito et al. & 2011 & 53 & 22 & 53 & 22 & $\mathrm{~S}, \mathrm{H}, \mathrm{U}$ \\
\hline Khoshbaten et al. & 2011 & 58 & 42 & 83 & 17 & S \\
\hline Murphy et al. & 2012 & 64 & 18 & 63 & 19 & S \\
\hline Xue et al. & 2013 & 7 & 3 & 988 & 503 & $S$ \\
\hline Obayo et al. & 2015 & 14 & 3 & 69 & 5 & $\mathrm{U}$ \\
\hline Poyrazoglu et al. & 2017 & 66 & 30 & 128 & 23 & $\mathrm{U}$ \\
\hline Tseng et al. & 2017 & 12 & 25 & 3,638 & 12,541 & $\mathrm{~S}, \mathrm{H}, \mathrm{U}$ \\
\hline Vohlonen et al. & 2018 & 6 & 14 & 6,178 & 5,232 & S \\
\hline
\end{tabular}

Note: Hp: Helicobacter pylori; S: serology; H: histology; U: rapid urease test.

(b) The data of Helicobacter pylori infection in esophageal adenocarcinoma

\begin{tabular}{|c|c|c|c|c|c|c|}
\hline \multirow{3}{*}{ First author } & \multirow{3}{*}{ Year } & \multicolumn{4}{|c|}{ Esophageal adenocarcinoma } & \multirow{3}{*}{$\begin{array}{c}\text { Hp } \\
\text { Test } \\
\text { method }\end{array}$} \\
\hline & & \multicolumn{2}{|c|}{ Case } & \multicolumn{2}{|c|}{ Control } & \\
\hline & & $\begin{array}{c}\mathrm{Hp} \\
+\end{array}$ & $\mathrm{Hp}-$ & $\mathrm{Hp}+$ & Hp- & \\
\hline Chow et al. & 1998 & 38 & 91 & 86 & 137 & $S$ \\
\hline Grimley et al. & 1999 & 24 & 16 & 25 & 21 & S \\
\hline Öberg et al. & 1999 & 5 & 32 & 32 & 197 & $\mathrm{H}$ \\
\hline Peek et al. & 1999 & 11 & 19 & 20 & 28 & $\mathrm{~S}, \mathrm{H}$ \\
\hline Vieth et al. & 2000 & 66 & 72 & 468 & 244 & $\mathrm{H}$ \\
\hline Weston et al. & 2000 & 3 & 17 & 96 & 121 & $\mathrm{H}$ \\
\hline Henrik et al. & 2001 & 0 & 7 & 67 & 82 & $S$ \\
\hline Wu et al. & 2003 & 49 & 31 & 230 & 126 & S \\
\hline El-Omar et al. & 2003 & 35 & 73 & 84 & 126 & S \\
\hline Ye et al. & 2004 & 18 & 79 & 198 & 301 & S \\
\hline De Martel et al. & 2005 & 19 & 32 & 74 & 76 & $\mathrm{~S}$ \\
\hline $\begin{array}{l}\text { Anandasabapathy } \\
\text { et al. }\end{array}$ & 2007 & 4 & 21 & 10 & 20 & $\mathrm{H}$ \\
\hline Simán et al. & 2007 & 4 & 8 & 24 & 23 & S \\
\hline Anderson et al. & 2008 & 55 & 68 & 157 & 96 & S \\
\hline
\end{tabular}

TABle 2: Continued.

\begin{tabular}{|c|c|c|c|c|c|c|}
\hline \multirow{3}{*}{ First author } & \multirow{3}{*}{ Year } & \multicolumn{4}{|c|}{ Esophageal adenocarcinoma } & \multirow{3}{*}{$\begin{array}{c}\text { Hp } \\
\text { Test } \\
\text { method }\end{array}$} \\
\hline & & \multicolumn{2}{|c|}{ Case } & \multicolumn{2}{|c|}{ Control } & \\
\hline & & $\begin{array}{c}\mathrm{Hp} \\
+\end{array}$ & Hp- & $\mathrm{Hp}+$ & Hp- & \\
\hline Früh et al. & 2008 & 36 & 64 & 43 & 58 & S \\
\hline Löfdahl et al. & 2008 & 130 & 100 & 304 & 195 & S \\
\hline Derakhshan et al. & 2008 & 9 & 10 & 28 & 10 & $S$ \\
\hline Whiteman et al. & 2010 & 35 & 225 & 302 & 1,014 & S \\
\hline Sonnenberg et al. & 2017 & 13 & 6,029 & 20,683 & 263,869 & $\mathrm{H}$ \\
\hline Vohlonen et al. & 2018 & 3 & 11 & 6,178 & 5,232 & S \\
\hline
\end{tabular}

Note: Hp: Helicobacter pylori; S: serology; H: histology; U: rapid urease test.

$P=0.462$, Europe: $P=0.461$, East Asia: $P=0.533$, and Middle East: $P=0.039)$, study type $(P=0.510)$, study object $(P=0.226)$, matching $(P=0.959)$, and detection method (serology: $P=0.139$; serology, histology, and rapid urease test: $P=0.052$ ) were assessed independently. In esophageal adenocarcinoma, year $(P=0.022)$, ethnicity (Caucasians: $P=0.009$, Australian: $P=0.135$ ), study area (North America: $P=0.717$, Europe: $P=0.853$, Middle East: $P=0.557)$, study type $(P=0.245)$, study object $(P=0.512)$, matching $(P=0.073)$, and detection method (serology: $P=0.626$, histology: $P=0.207$ ) were also assessed independently. The results of the univariate analysis were presented in Table 4. If the regression coefficient of the covariate was significant at the level of 0.1 , then the covariate was entered into the multivariate metaregression. In esophageal squamous cell carcinoma, the study area (North America: $P=0.455$, Europe: $P=0.368$, East Asia: $P=0.395$, Middle East: $P=0.05$ ) and detection method (serology: $P=0.671$; serology, histology, and rapid urease test: $P=0.752$ ) were assessed simultaneously. In esophageal adenocarcinoma, year $(P=0.49)$, ethnicity (Caucasians: $P=0.361$, Australian: $P=0.306)$, and matching $(P=0.209)$ were also assessed simultaneously. The results of the multivariate analysis were presented in Table 5.

\section{Discussion}

This meta-analysis was based on 35 studies with 345,886 patients, which is much larger than the previous data. Our meta-analysis showed no significant correlation between Helicobacter pylori infection and esophageal squamous cell carcinoma in the general population. Some studies have reported similar results $[36,43]$. However, some researchers believed that Helicobacter pylori infection may play a protective role in the risk of esophageal squamous cell carcinoma $[37,47]$. Another result of our meta-analysis seemed to explain this inconsistency. The risk of esophageal squamous cell carcinoma varies from region to region. Due to the considerable heterogeneity, we also use the HKSJ method for statistics. Compared to the nonsignificant difference in other regions, lower risk of esophageal squamous cell carcinoma was found in the Middle East. It is different from the DL method whose result showed no increase of the risk in North America. But some scholars believed that Helicobacter 
Table 3

(a) Subgroup comparisons for Helicobacter pylori infection on the risk of esophageal squamous cell carcinoma

\begin{tabular}{|c|c|c|c|c|c|}
\hline \multirow{3}{*}{$\begin{array}{l}\text { Subgroup } \\
\text { Esophageal squamous cell carcinoma }\end{array}$} & \multirow{3}{*}{ No. of studies } & \multirow{3}{*}{$I^{2}(\%)$} & \multicolumn{2}{|c|}{ Overall OR (95\% CI) } & \multirow{3}{*}{$P$ value } \\
\hline & & & DL & HKSJ & \\
\hline & & & & & \\
\hline \multicolumn{6}{|l|}{ Case/control $(2,063 / 35,051)$} \\
\hline All studies & 21 & 78.5 & $0.84(0.64,1.09)$ & $0.74(0.54,0.97)$ & \\
\hline Ethnicity & & & & & 0.144 \\
\hline Caucasians & 11 & 70.8 & $0.80(0.54,1.17)$ & $0.73(0.48,1.11)$ & \\
\hline Mongolians & 8 & 86.9 & $0.89(0.56,1.41)$ & $1.13(0.79,1.60)$ & \\
\hline Negroes & 1 & - & $0.34(0.07,1.58)$ & - & \\
\hline Australian & 1 & - & $1.18(0.84,1.65)$ & - & \\
\hline Study area & & & & & $<0.001^{*}$ \\
\hline North America & 2 & 0 & $1.83(1.17,2.87)$ & $1.79(0.25,12.9)$ & \\
\hline East Asia & 8 & 86.9 & $0.89(0.56,1.41)$ & $1.13(0.79,1.60)$ & \\
\hline Europe & 7 & 0.9 & $0.84(0.65,1.10)$ & $0.72(0.46,1.12)$ & \\
\hline Middle East & 3 & 0 & $0.34(0.22,0.52)$ & $0.34(0.26,0.44)$ & \\
\hline Oceania & 1 & - & $1.18(0.84,1.65)$ & - & \\
\hline Study type & & & & & 0.250 \\
\hline Case-control & 19 & 80 & $0.85(0.65,1.13)$ & $0.70(0.51,0.97)$ & \\
\hline Cohort & 2 & 49 & $0.59(0.19,1.86)$ & $0.80(0.0,967.18)$ & \\
\hline Study object & & & & & $0.005^{*}$ \\
\hline Population-based studies & 14 & 79.5 & $0.93(0.68,1.28)$ & $0.91(0.66,1.24)$ & \\
\hline Clinical-based studies & 7 & 72.5 & $0.66(0.40,1.07)$ & $0.53(0.28,0.99)$ & \\
\hline Matching & & & & & 0.290 \\
\hline Studies with matched controls & 2 & 86.5 & $0.87(0.30,2.52)$ & $1.14(0.0,563.04)$ & \\
\hline Studies without matched controls & 19 & 78.7 & $0.83(0.63,1.11)$ & $0.71(0.52,0.96)$ & \\
\hline Helicobacter pylori detection method & & & & & $0.003^{*}$ \\
\hline Serology & 16 & 81.4 & $0.83(0.61,1.12)$ & $0.83(0.61,1.14)$ & \\
\hline Rapid urease test & 2 & 0 & $0.39(0.22,0.69)$ & $0.35(0.17,0.69)$ & \\
\hline Serology, histology, and rapid urease test & 3 & 0 & $1.32(0.87,2.01)$ & $1.33(0.72,2.44)$ & \\
\hline
\end{tabular}

Note: DL: DerSimonian-Laird; HKSJ: Hartung-Knapp-Sidik-Jonkman. ${ }^{\S}$ Differences between subgroups; ${ }^{*}$ the difference was statistically significant.

(b) Subgroup comparisons for Helicobacter pylori infection on the risk of esophageal adenocarcinoma

\begin{tabular}{|c|c|c|c|c|c|}
\hline \multirow{2}{*}{ Subgroup } & \multirow{3}{*}{ No. of studies } & \multirow{3}{*}{$I^{2}(\%)$} & \multicolumn{2}{|c|}{ Overall OR (95\% CI) } & \multirow{3}{*}{$P$ value ${ }^{\S}$} \\
\hline & & & DL & HKSJ & \\
\hline \multicolumn{3}{|c|}{ Esophageal adenocarcinoma } & & & \\
\hline \multicolumn{6}{|c|}{ Case/control (7687/301085) } \\
\hline All studies & 20 & 73.4 & $0.55(0.43,0.70)$ & $0.23(0.15,0.36)$ & \\
\hline Ethnicity & & & & & $<0.001^{*}$ \\
\hline Caucasians & 18 & 33.9 & $0.60(0.50,0.73)$ & $0.23(0.14,0.37)$ & \\
\hline Mixed race & 1 & - & $0.29(0.25,0.35)$ & - & \\
\hline Australian & 1 & - & $0.52(0.36,0.76)$ & - & \\
\hline Study area & & & & & 0.100 \\
\hline North America & 11 & 80.5 & $0.62(0.42,0.93)$ & $0.54(0.35,0.81)$ & \\
\hline Europe & 7 & 56.2 & $0.49(0.34,0.69)$ & $0.13(0.06,0.27)$ & \\
\hline Middle East & 1 & - & $0.32(0.10,1.02)$ & - & \\
\hline Oceania & 1 & - & $0.52(0.36,0.76)$ & - & \\
\hline Study type & & & & & 0.287 \\
\hline Case-control & 19 & 74.4 & $0.56(0.44,0.72)$ & $0.23(0.14,0.37)$ & \\
\hline
\end{tabular}


TABLE 3: Continued.

\begin{tabular}{|c|c|c|c|c|c|}
\hline \multirow{2}{*}{ Subgroup } & \multirow{2}{*}{ No. of studies } & \multirow{2}{*}{$I^{2}(\%)$} & \multicolumn{2}{|c|}{ Overall OR $(95 \% \mathrm{CI})$} & \multirow{3}{*}{$P$ value } \\
\hline & & & DL & HKSJ & \\
\hline \multicolumn{5}{|l|}{ Esophageal adenocarcinoma } & \\
\hline Cohort & 1 & - & $0.23(0.06,0.83)$ & - & \\
\hline Study object & & & & & $<0.001^{*}$ \\
\hline Population-based studies & 11 & 37.9 & $0.59(0.48,0.73)$ & $0.15(0.08,0.28)$ & \\
\hline Clinical-based studies & 9 & 73.4 & $0.52(0.35,0.79)$ & $0.47(0.29,0.77)$ & \\
\hline Matching & & & & & $<0.001^{*}$ \\
\hline Studies with matched controls & 5 & 0 & $0.73(0.57,0.92)$ & $0.71(0.60,0.84)$ & \\
\hline Studies without matched controls & 15 & 73.3 & $0.48(0.36,0.65)$ & $0.21(0.13,0.36)$ & \\
\hline Helicobacter pylori detection method & & & & & $<0.001^{*}$ \\
\hline Serology & 14 & 36.3 & $0.62(0.51,0.75)$ & $0.19(0.11,0.32)$ & \\
\hline Histology & 5 & 62.1 & $0.39(0.26,0.60)$ & $0.39(0.18,0.84)$ & \\
\hline Serology, histology & 1 & - & $0.81(0.32,2.07)$ & - & \\
\hline
\end{tabular}

Note: DL: DerSimonian-Laird; HKSJ: Hartung-Knapp-Sidik-Jonkman. ${ }^{\circledR}$ Differences between subgroups; * the difference was statistically significant.

pylori infection can cause esophageal squamous cell carcinoma through gastric atrophy which may promote the excessive growth of bacteria and increase the production of endogenous nitrosamines, then lead to the esophageal squamous cell carcinoma $[49,57,58]$. However, ESCC is more common in nonindustrialized countries. Smoking and alcohol consumption are the main risk factors which have been proven to be associated with a multiplied risk of the development of esophageal cancer in Western countries $[59,60]$. But no significant impact of the two factors were found in northern Iran $[61,62]$. The difference of risk factors in different regions may result in the different relationships. Moreover, this difference may also be caused by different dietary cultures in different regions. Multiple detection methods can reduce the false negative of Helicobacter pylori diagnosis. Our results showed that different detection methods were statistically significant that the influence of the false negative cannot be excluded. Besides, some studies pointed that Helicobacter pylori may spontaneously disappear with a progression of gastric atrophy or metaplasia, which leads to false negative and may also have a potential impact on the results $[63,64]$.

As for esophageal adenocarcinoma, we found that Helicobacter pylori infection may reduce its risk, which is consistent with several previous meta-analyses $[4,14,15$, 65]. At the same time, the Helicobacter pylori infection rate has decreased year by year. There are currently more reliable assumptions about this phenomenon: (1) Helicobacter pylori infection, accompanied by atrophy of gastric body and loss of parietal cells, resulting in reduced reflux, which reduces the incidence of reflux esophagitis and Barrett's esophagus [66]; (2) Helicobacter pylori infection can induce apoptosis of esophageal adenocarcinoma cells progressing from Barrett's esophagus through Fas apoptotic pathway mediated by Caspase [60]. But there are also some other claims that Helicobacter pylori infection is a risk factor for esophageal adenocarcinoma. On the one hand, gastrin induced by Helicobacter pylori is a carcinogenic growth factor, which contributes to the canceration of the esophagus and stomach, especially playing a potential causal role in the progression of Barrett's esophageal neoplasm. On the other hand, Helicobacter pylori induces the expression of nuclear factor-kappa $\mathrm{B}(\mathrm{NF}-\kappa \mathrm{B})$ and cyclooxygenase- (COX-) 2 in esophageal epithelial cells and plays a role in the inflammation associated with Barrett's esophagus and tumorigenesis in the esophagus [59]. Studies have found that the prevalence of esophageal adenocarcinoma with persistent Helicobacter pylori infection is higher than that after eradication therapy [62]. When matching the control group and the case group, the two different statistical methods showed that the pooled OR of the matching group was significantly higher than that of the nonmatching group. The protective effect of Helicobacter pylori on adenocarcinoma was not so obvious, and there were significant differences between the groups $(P<0.001)$. The incidence of esophageal adenocarcinoma increases with age, and there is male predominance in esophageal adenocarcinoma. The incidence of esophageal adenocarcinoma in men and women is $6: 1$ in general, which is as high as $8: 1$ in some of the other populations, such as in the United States $[61,67]$. Therefore, the matching of the case group and the control group may make a great difference in the results. Our meta-analysis showed that the prevalence of Helicobacter pylori was almost the same between the case group and the control group. Take et al. [68] conducted a 20-year study of 2,782 patients and concluded that the risk of esophageal adenocarcinoma caused by Helicobacter pylori eradication may be unfounded. Besides, epidemiological studies have shown that the incidence of gastroesophageal reflux disease, Barrett's esophagus, and distal esophageal cancer is lower in Malaysians with lower prevalence of Helicobacter pylori infection [69]. Moreover, we grouped the detection methods of Helicobacter pylori and found that the pooled OR of multiple detection methods was 0.81 (95\% CI: 0.32-2.07). A variety of detection methods can minimize false negative as far as possible. Due to the limited number of the articles, further research needs to be performed in the future. 
Study

ID

$\%$

OR (95\% CI) weight

Caucasians

Talley et al. (1991)

Henrik et al. (2001)

El-Omar et al. (2003)

Ye et al. (2004)

Siman et al. (2007)

CooK et al. (2010)

Venerito et al. (2011)

Khoshbaten et al. (2011)

Murphy et al. (2012)

Poyrazoglu et al. (2017)

Vohlonen et al. (2018)

Subtotal $(I$-squared $=70.8 \%, P=0.000)$

.

Mongolians

Wang et al. (2003)

Wu et al. (2005)

Kamangar et al. (2007)

Iijima et al. (2007)

Wu et al. (2009)

Hu et al. (2009)

Xue et al. (2013)

Tseng et al. (2017)

Subtotal $(I$-squared $=86.9 \%, P=0.000)$

.

Australian

Whiteman et al. (2010)

Subtotal $(I$-squared $=. \%, P=$.)

Negroes

Obayo et al. (2015)

Subtotal $(I$-squared $=. \%, P=$. $)$

.

Overall $(I$-squared $=78.5 \%, P=0.000)$

Note: weights are from random effects analysis

.0723

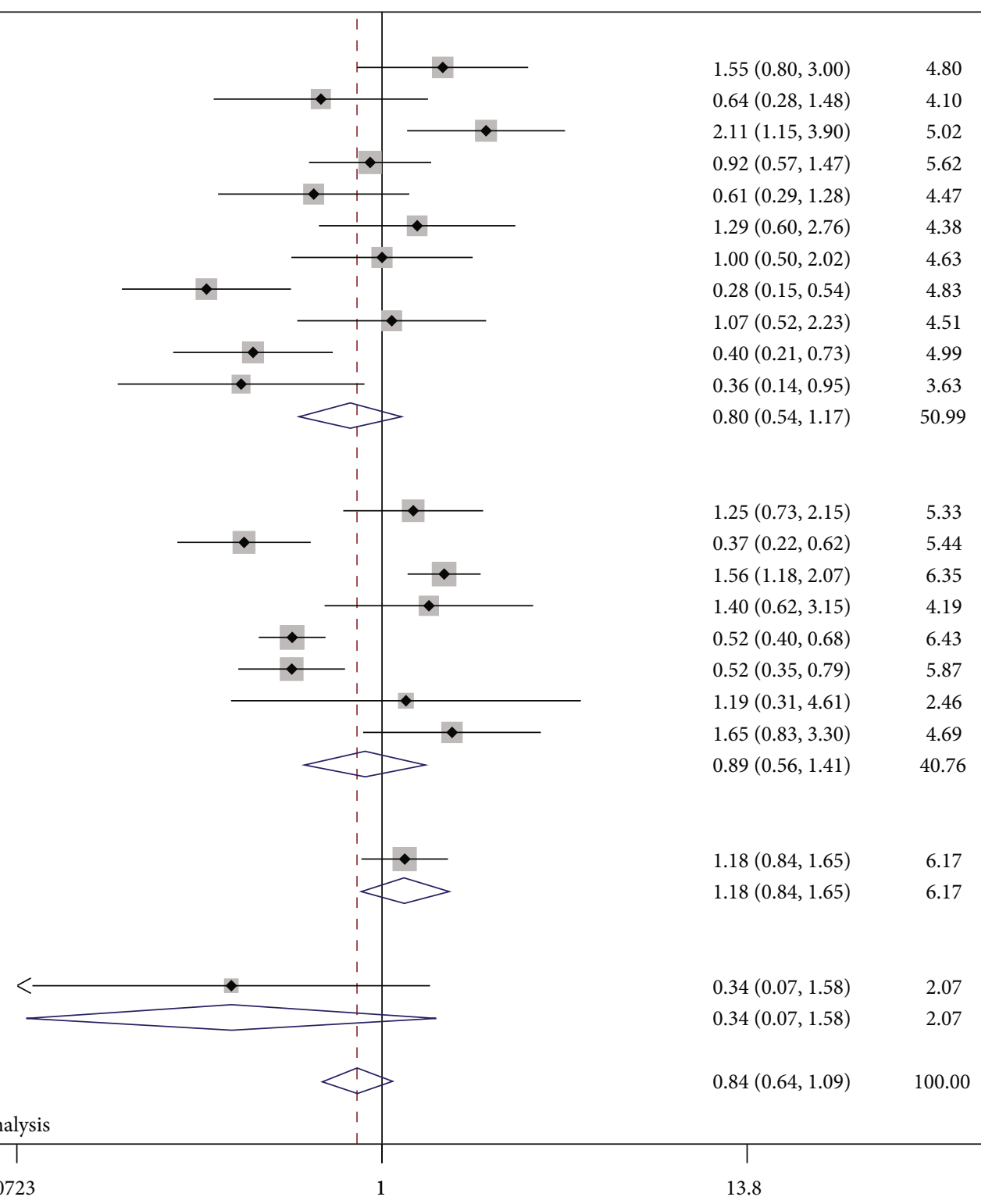

(a)

FIgure 2: Continued. 


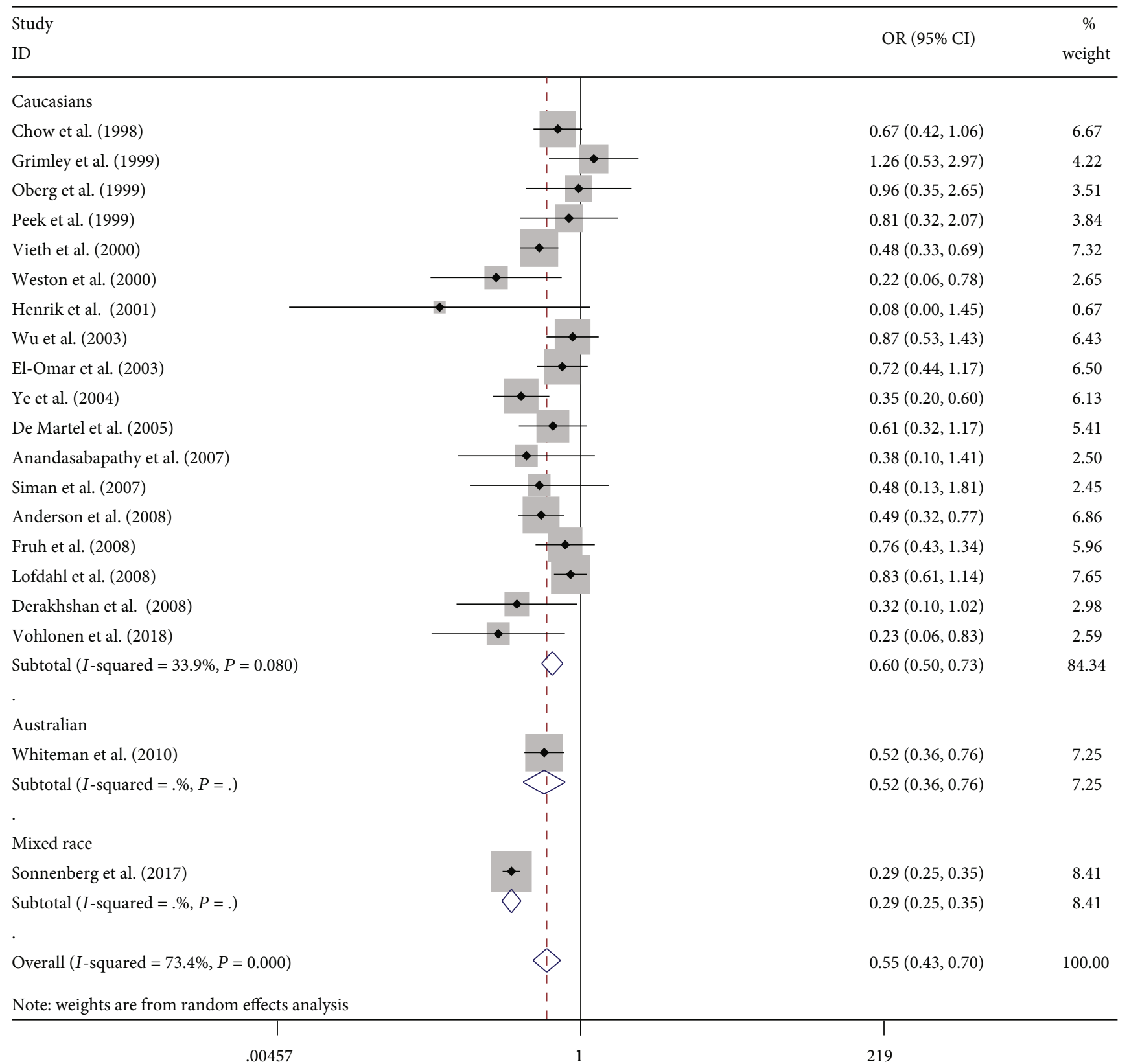

(b)

FIGURE 2: Meta-analysis of the association between Helicobacter pylori infection and esophageal cancer after grouping based on ethnicity: (a) forest plot of esophageal squamous cell carcinoma; (b) forest plot of esophageal adenocarcinoma. Each horizontal bar summarizes a study. Bars represent 95\% CIs. Gray squares inform on each of the studies' weight in the meta-analysis. Diamond in the lower part of the graph depicts the pooled estimate along with $95 \%$ CIs.

Although current evidence suggests that Helicobacter pylori infection may reduce the risk of esophageal adenocarcinoma, this claim may be one-sided, and the "protective effect" of Helicobacter pylori infection may be overestimated. We found that there were significant statistical differences among ethnicities. This might be because the etiology of esophageal adenocarcinoma is related to the genetic factors and is attributed to the mutations in the lineage [70].

Heterogeneity might affect the interpretation of the results. This meta-analysis showed that there was consid- erable heterogeneity. Regarding esophageal squamous cell carcinoma, we performed a subgroup analysis of six possible factors. It was found that when grouped according to the study area, study type, and detection of Helicobacter pylori, heterogeneity decreased significantly. We then conducted metaregression and found that the study area may contribute more to the overall heterogeneity. As we have found, the risk of esophageal squamous cell carcinoma varies from area to area. In esophageal adenocarcinoma, subgroup analysis demonstrated that the heterogeneity was significantly lower 


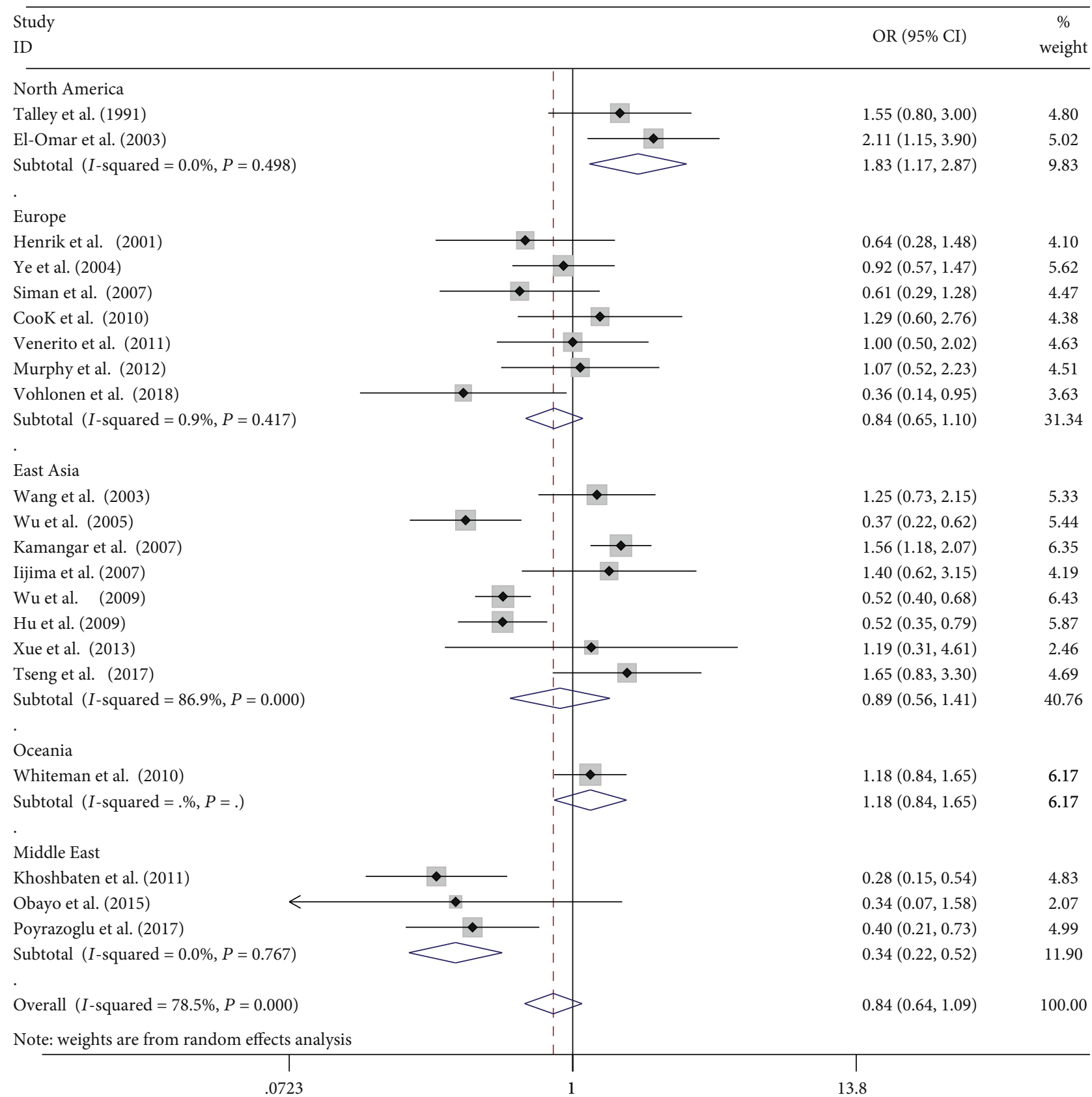

(a)

Figure 3: Continued. 


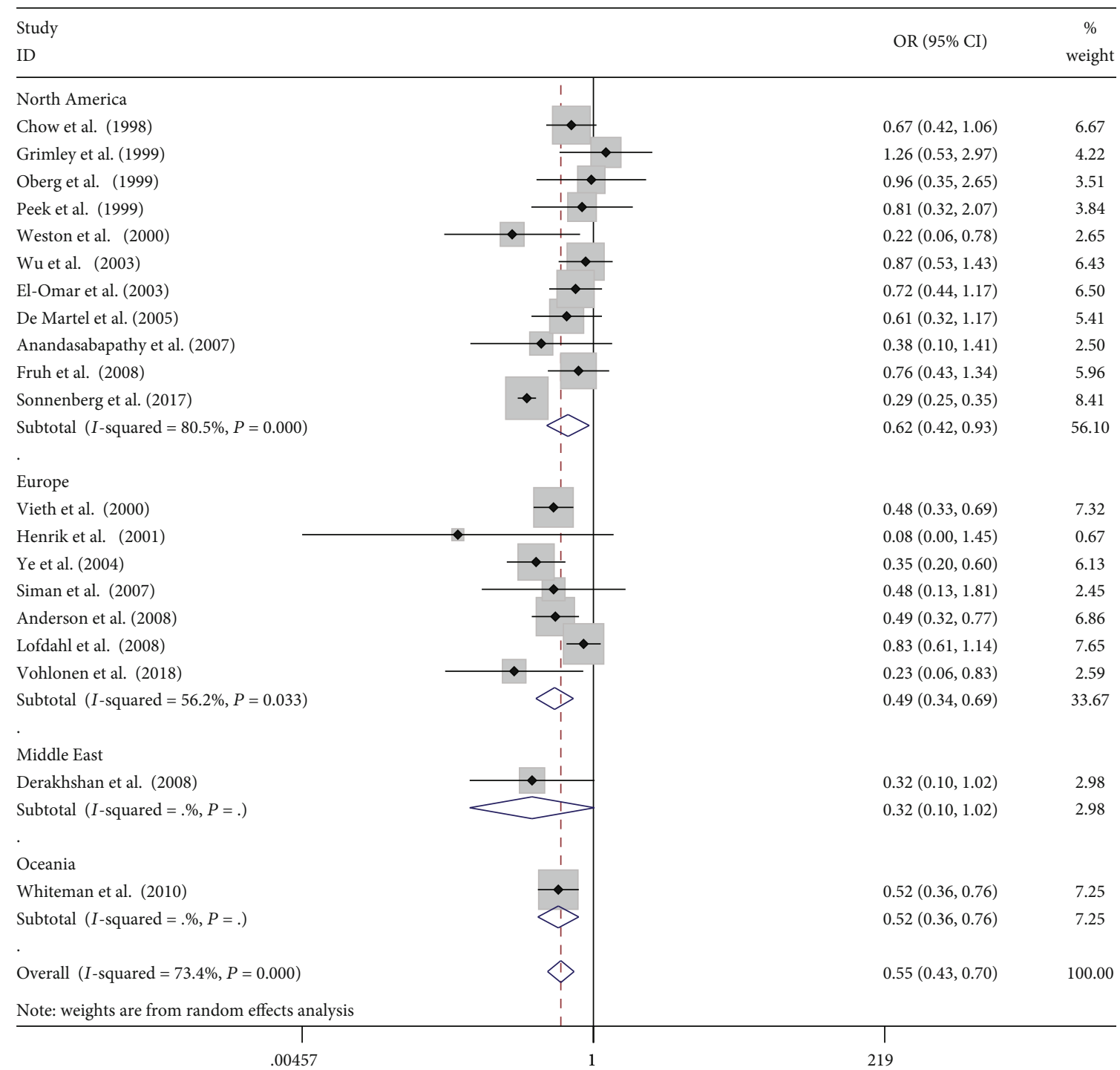

(b)

FIgURE 3: Meta-analysis of the association between Helicobacter pylori infection and esophageal cancer after grouping based on region: (a) forest plot of esophageal squamous cell carcinoma; (b) forest plot of esophageal adenocarcinoma. Each horizontal bar summarizes a study. Bars represent 95\% CIs. Gray squares inform on each of the studies' weight in the meta-analysis. Diamond in the lower part of the graph depicts the pooled estimate along with $95 \%$ CIs.

Funnel plot with pseudo 95\% confidence limits

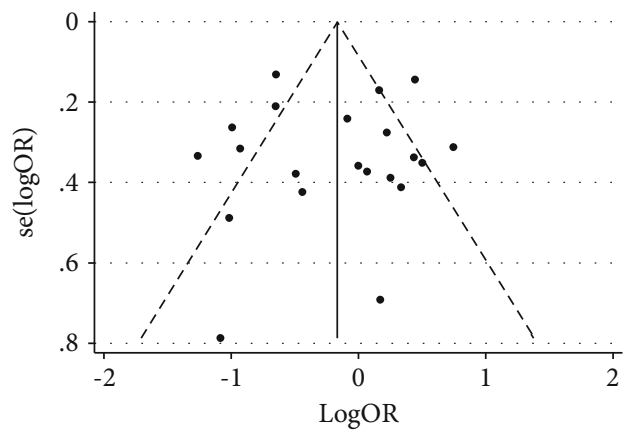

(a)

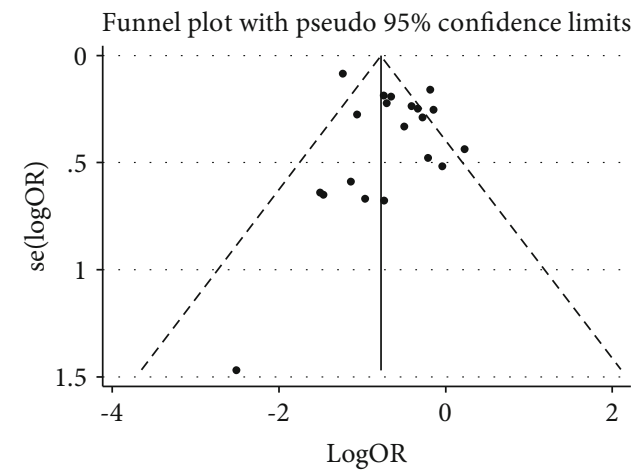

(b)

FIGURE 4: Funnel plot of the association between Helicobacter pylori infection and esophageal cancer: (a) esophageal squamous cell carcinoma; (b) esophageal adenocarcinoma. 
TABLE 4: Univariate metaregression analysis for the potential variables between studies.

\begin{tabular}{|c|c|c|c|c|c|c|}
\hline \multirow{2}{*}{$\begin{array}{l}\text { Covariates } \\
\text { ESCC }\end{array}$} & \multirow[t]{2}{*}{ Coefficient } & \multirow[t]{2}{*}{ Standard error } & \multirow[t]{2}{*}{$t$} & \multirow[t]{2}{*}{$P$} & \multicolumn{2}{|c|}{$95 \%$ confidence interval } \\
\hline & & & & & & \\
\hline Year & -0.034 & 0.022 & -1.56 & 0.135 & -0.080 & 0.011 \\
\hline \multicolumn{7}{|l|}{ Ethnicity } \\
\hline Caucasians & 0.855 & 0.977 & 0.88 & 0.394 & -1.206 & 2.917 \\
\hline Mongolians & 0.961 & 0.982 & 0.98 & 0.341 & -1.111 & 3.033 \\
\hline Australian & 1.247 & 1.114 & 1.12 & 0.279 & -1.103 & 3.598 \\
\hline \multicolumn{7}{|l|}{ Study area } \\
\hline North America & 0.434 & 0.577 & 0.75 & 0.462 & -0.788 & 1.656 \\
\hline Europe & -0.370 & 0.490 & -0.76 & 0.461 & -1.408 & 0.669 \\
\hline East Asia & -0.304 & 0.477 & -0.64 & 0.533 & -1.315 & 0.707 \\
\hline Middle East & -1.254 & 0.559 & -2.24 & $0.039^{*}$ & -2.439 & -0.692 \\
\hline Study type & 0.379 & 0.564 & 0.67 & 0.510 & -0.803 & 1.560 \\
\hline Study object & 0.356 & 0.284 & 1.25 & 0.226 & -0.239 & 0.950 \\
\hline Matching & 0.024 & 0.447 & 0.05 & 0.959 & -0.911 & 0.959 \\
\hline \multicolumn{7}{|l|}{ Detection method } \\
\hline $\mathrm{S}$ & 0.785 & 0.508 & 1.55 & 0.139 & -0.281 & 1.852 \\
\hline $\mathrm{S}, \mathrm{H}, \mathrm{U}$ & 1.251 & 0.600 & 2.08 & 0.052 & -0.010 & 2.512 \\
\hline \multicolumn{7}{|l|}{ EAC } \\
\hline Year & -0.040 & 0.016 & -2.50 & $0.022^{*}$ & -0.073 & -0.006 \\
\hline \multicolumn{7}{|l|}{ Ethnicity } \\
\hline Caucasians & 0.729 & 0.249 & 2.93 & $0.009^{*}$ & 0.204 & 1.254 \\
\hline Australian & 0.581 & 0.370 & 1.57 & 0.135 & -0.199 & 1.362 \\
\hline \multicolumn{7}{|l|}{ Study area } \\
\hline North America & 0.163 & 0.442 & 0.37 & 0.717 & -0.774 & 1.100 \\
\hline Europe & -0.866 & 0.459 & -0.19 & 0.853 & -1.059 & 0.885 \\
\hline Middle East & -0.485 & 0.808 & -0.60 & 0.557 & -2.199 & 1.228 \\
\hline Study type & 0.883 & 0.735 & 1.20 & 0.245 & -0.661 & 2.426 \\
\hline Study object & -0.142 & 0.212 & -0.67 & 0.512 & -0.587 & 0.304 \\
\hline Matching & 0.404 & 0.212 & 1.90 & 0.073 & -0.421 & 0.849 \\
\hline \multicolumn{7}{|l|}{ Detection method } \\
\hline S & -0.278 & 0.560 & -0.50 & 0.626 & -1.461 & 0.903 \\
\hline $\mathrm{H}$ & -0.757 & 0.577 & -1.31 & 0.207 & -1.974 & 0.460 \\
\hline
\end{tabular}

Note: ESCC: esophageal squamous cell carcinoma; EAC: esophageal adenocarcinoma; S: serology; H: histology; U: rapid urease test. *the difference was statistically significant.

after grouping by ethnicity, study object, matching, and detection of Helicobacter pylori. Metaregression showed that matching might contribute little to the overall heterogeneity; then, year and ethnicity may contribute more to the overall heterogeneity. However, multivariate regression analysis showed that year and ethnicity did not significantly differ. This may be related to the annual concentration of the articles we have included based on ethnicity.

The advantages of our meta-analysis are as follows: Firstly, compared to the previous meta-analyses, our study included cohort studies, and the number of enrolled studies was more reliable. Subgroup analysis and metaregression were conducted to identify potential sources of heterogeneity; then, this study analyzed whether the differences between the groups were statistically significant. Secondly, all the studies in our meta-analysis have acceptable quality, the results of sensitivity analysis are stable, and the impact of publication bias is small. Thirdly, we analyzed the relationship between Helicobacter pylori infection and the risk of esophageal cancer in more details, whether different ethnicities and regions are the same and whether the differences have statistical significance. However, this study still has limitations. Firstly, this meta-analysis has not been registered online, which may cause bias in the analysis. Secondly, a special disadvantage of most original studies is the confounding factor; that is, many factors are not taken into account in research design or data analysis. Moreover, current epidemiological studies provide uncertain data on the negative or neutral correlation between Helicobacter pylori infection and esophageal adenocarcinoma. The analysis of observational studies supports the negative correlation that we have not analyzed the differences. Finally, the 
TABLE 5: Multivariate metaregression analysis for the potential variables between studies.

\begin{tabular}{|c|c|c|c|c|c|c|}
\hline Covariates & Coefficient & Standard error & $t$ & $P$ & \multicolumn{2}{|c|}{$95 \%$ confidence interva } \\
\hline \multicolumn{7}{|l|}{ ESCC } \\
\hline \multicolumn{7}{|l|}{ Study area } \\
\hline North America & 0.434 & 0.566 & 0.77 & 0.455 & -0.779 & 1.647 \\
\hline Europe & 0.449 & 0.483 & -0.93 & 0.368 & -1.485 & 0.586 \\
\hline East Asia & -0.414 & 0.472 & -0.88 & 0.395 & -1.427 & 0.599 \\
\hline Middle East & -1.426 & 0.677 & -2.11 & $0.05^{*}$ & -2.878 & 0.026 \\
\hline \multicolumn{7}{|l|}{ Detection method } \\
\hline S & -0.296 & 0.681 & -0.43 & 0.671 & -1.757 & 1.165 \\
\hline $\mathrm{S}, \mathrm{H}, \mathrm{U}$ & 0.246 & 0.765 & 0.32 & 0.752 & -1.395 & 1.888 \\
\hline \multicolumn{7}{|l|}{ EAC } \\
\hline Year & -0.017 & 0.024 & -0.71 & 0.490 & -0.069 & -0.035 \\
\hline \multicolumn{7}{|l|}{ Ethnicity } \\
\hline Caucasians & 0.394 & 0.417 & 0.94 & 0.361 & -0.496 & 1.283 \\
\hline Australian & 0.461 & 0.435 & 1.06 & 0.306 & -0.467 & 1.389 \\
\hline Matching & 0.275 & 0.209 & 1.31 & 0.209 & -0.171 & 0.721 \\
\hline
\end{tabular}

Note: ESCC: esophageal squamous cell carcinoma; EAC: esophageal adenocarcinoma; S: serology; H: histology; U: rapid urease test. *the difference was statistically significant.

heterogeneity of the total combined effects of esophageal squamous cell carcinoma and esophageal adenocarcinoma is high in our study. Although these heterogeneities can be reduced after subgroup analysis, they might lead to a reduction in the number of studies and thus limit the reliability of data.

\section{Conclusions}

In summary, no significant association was found between Helicobacter pylori infection and the risk of esophageal squamous cell carcinoma in the general population. However, lower risk was found in the Middle East when grouped by the study area. In the ethnic stratification analysis, there was no significant correlation between Helicobacter pylori infection and the risk of esophageal squamous cell carcinoma. Helicobacter pylori infection may reduce the risk of esophageal adenocarcinoma in the general population, but this may be one-sided; the statement of "protection effect" may be overestimated. Therefore, well-designed prospective cohort studies with a powered sample size are required, in which potential confounders should be taken into account to validate their relationship.

\section{Appendix}

The following are the PubMed Search Terms: ("Esophageal Neoplasm" [MeSH] or "Neoplasm, Esophageal" [MeSH] or "Esophagus Neoplasm" [MeSH] or "Esophagus Neoplasms" [MeSH] or "Neoplasm, Esophagus" [MeSH] or "Neoplasms, Esophagus" [MeSH] or "Neoplasms, Esophageal" [MeSH] or "Esophageal Neoplasms" [MeSH] or "Cancer of Esophagus" [MeSH] or "Cancer of the Esophagus" [MeSH] or "Esophagus Cancer" [MeSH] or "Cancer, Esophagus" [MeSH] or "Cancers, Esophagus" [MeSH] or "Esophagus Cancers" $[\mathrm{MeSH}]$ or "Esophageal Cancer" $[\mathrm{MeSH}]$ or "Cancer,
Esophageal" [MeSH] or "Cancers, Esophageal" [MeSH] or "Esophageal Cancers" [MeSH] or "esophageal carcinoma" $[\mathrm{MeSH}]$ or "esophageal carcinomas" $[\mathrm{MeSH}]$ or "Esophagus carcinoma" $[\mathrm{MeSH}]$ or "Esophagus carcinomas" [MeSH] or "esophageal tumor" [MeSH] or "esophageal tumors" [MeSH] or "Esophagus tumors" [MeSH] or "Esophagus tumor" [MeSH] or "esophageal squamous cell carcinoma" [MeSH] or "ESCC" [MeSH] or "EAC" [All Fields] or "esophageal squamous carcinoma" [MeSH] or "esophageal adenocarcinoma" $[\mathrm{MeSH}]$ or "adenocarcinoma of the esophagus" $[\mathrm{MeSH}]$ ) and ("Helicobacter pylori" $[\mathrm{MeSH}]$ or "H pylori" [MeSH] or "H. pylori" [MeSH] or "HP" [All Fields] or "Helicobacter" [MeSH]).

The following are the Embase Search Terms: ("Esophageal Neoplasm" [Emtree] or "Neoplasm, Esophageal" [Emtree] or "Esophagus Neoplasm" [Emtree] or "Esophagus Neoplasms" [Emtree] or "Neoplasm, Esophagus" [Emtree] or "Neoplasms, Esophagus" [Emtree] or "Neoplasms, Esophageal" [Emtree] or "Esophageal Neoplasms" [Emtree] or "Cancer of Esophagus" [Emtree] or "Cancer of the Esophagus" [Emtree] or "Esophagus Cancer" [Emtree] or "Cancer, Esophagus" [Emtree] or "Cancers, Esophagus" [Emtree] or "Esophagus Cancers" [Emtree] or "Esophageal Cancer" [Emtree] or "Cancer, Esophageal" [Emtree] or "Cancers, Esophageal" [Emtree] or "Esophageal Cancers" [Emtree] or "esophageal carcinoma" [Emtree] or "esophageal carcinomas" [Emtree] or "Esophagus carcinoma" [Emtree] or "Esophagus carcinomas" [Emtree] or "esophageal tumor" [Emtree] or "esophageal tumors" [Emtree] or "Esophagus tumors" [Emtree] or "Esophagus tumor" [Emtree] or "esophageal squamous cell carcinoma" [Emtree] or "ESCC" [Emtree] or "EAC” [Emtree] or "esophageal squamous carcinoma" [Emtree] or "esophageal adenocarcinoma" [Emtree] or "adenocarcinoma of the esophagus" [Emtree]) and ("Helicobacter pylori" [Emtree] or "H pylori" [Emtree] or "H. pylori" [Emtree] or "HP" [Emtree] or "Helicobacter" [Emtree]). 


$\begin{array}{ll}\text { Abbreviations } \\ \text { ESCC: } & \text { Esophageal squamous cell carcinoma } \\ \text { EAC: } & \text { Esophageal adenocarcinoma } \\ \text { HP/H. pylori: } & \text { Helicobacter pylori } \\ \text { MOOSE: } & \text { Meta-analysis of Observational Studies in } \\ & \text { Epidemiology } \\ \text { NOS: } & \text { The Newcastle-Ottawa Scale } \\ \text { OR: } & \text { Odds ratio } \\ \text { RR: } & \text { Relative risk } \\ \text { CI: } & \text { Confidence interval } \\ \text { NF- } \kappa \text { B: } & \text { Nuclear factor-kappa B } \\ \text { COX-2: } & \text { Cyclooxygenase-2 } \\ \text { DL: } & \text { DerSimonian-Laird } \\ \text { HKSJ: } & \text { Hartung-Knapp-Sidik-Jonkman. }\end{array}$

\section{Conflicts of Interest}

The authors declare that they have no competing interests.

\section{Authors' Contributions}

HG contributed to study design, literature search, quality assessment, data extraction and data analysis, manuscript drafting, and editing. JJ contributed to research identification and selection, data extraction, data analysis and interpretation, manuscript drafting, and editing. WP contributed to data analysis and interpretation, manuscript drafting, and editing. LL contributed to research identification and selection, data extraction and quality assessment, manuscript drafting, and editing. CZ contributed to the design and discussion of the study, manuscript drafting, and editing. JT, XG, JW, and XZ contributed to manuscript drafting and editing. All authors have approved the final version of this manuscript.

\section{Acknowledgments}

This study was supported by the Zhejiang Medicine Key Scientific and Technology Project (grant number: 2018258924) and Zhejiang Medicine Scientific and Technology Project (grant number: 2019RC094).

\section{Supplementary Materials}

Table S1: critical appraisal of the included studies according to the Newcastle-Ottawa Scale. Table S2: assessment of risk of bias in individual studies. Table S3: checklist for Metaanalysis of Observational Studies in Epidemiology (MOOSE). (Supplementary Materials)

\section{References}

[1] J. Ferlay, I. Soerjomataram, R. Dikshit et al., "Cancer incidence and mortality worldwide: sources, methods and major patterns in GLOBOCAN 2012," International Journal of Cancer, vol. 136, no. 5, pp. E359-E386, 2015.

[2] F. Turati, I. Tramacere, C. La Vecchia, and E. Negri, "A metaanalysis of body mass index and esophageal and gastric cardia adenocarcinoma," Annals of Oncology, vol. 24, no. 3, pp. 609617, 2013.

[3] I. Tramacere, C. La Vecchia, and E. Negri, “Tobacco smoking and esophageal and gastric cardia adenocarcinoma," Epidemiology, vol. 22, no. 3, pp. 344-349, 2011.

[4] J. H. Rubenstein and J. B. Taylor, "Meta-analysis: the association of oesophageal adenocarcinoma with symptoms of gastro-oesophageal reflux," Alimentary Pharmacology \& Therapeutics, vol. 32, no. 10, pp. 1222-1227, 2010.

[5] C. Y. Kao, B. S. Sheu, and J. J. Wu, "Helicobacter pylori infection: an overview of bacterial virulence factors and pathogenesis," Biomedical Journal, vol. 39, no. 1, pp. 1423, 2016.

[6] B. J. Marshall and J. R. Warren, "Unidentified curved bacilli in the stomach of patients with gastritis and peptic ulceration," The Lancet, vol. 1, no. 8390, pp. 1311-1315, 1984.

[7] IARC Working Group on the Evaluation of Carcinogenic Risks to Humans, "Biological agents. Volume 100 B. A review of human carcinogens," IARC Monographs on the Evaluation of Carcinogenic Risks to Humans, vol. 100, Part B, pp. 1-441, 2012.

[8] X. W. Wu, H. Z. Ji, M. F. Yang, L. Wu, and F. Y. Wang, "Helicobacter pylori infection and inflammatory bowel disease in Asians: a meta-analysis," World Journal of Gastroenterology, vol. 21, no. 15, pp. 4750-4756, 2015.

[9] I. Daugule, J. Zavoronkova, and D. Santare, "Helicobacter pylori and allergy: update of research," World Journal of Methodology, vol. 5, no. 4, pp. 203-211, 2015.

[10] N. Lender, N. J. Talley, P. Enck et al., "Review article: associations between Helicobacter pylori and obesity-an ecological study," Alimentary Pharmacology \& Therapeutics, vol. 40, no. 1, pp. 24-31, 2014.

[11] M. Grande, F. Cadeddu, M. Villa et al., "Helicobacter pylori and gastroesophageal reflux disease," World Journal of Surgical Oncology, vol. 6, no. 1, p. 74, 2008.

[12] S. Nie, T. Chen, X. Yang, P. Huai, and M. Lu, "Association of Helicobacter pylori infection with esophageal adenocarcinoma and squamous cell carcinoma: a meta-analysis," Diseases of the Esophagus, vol. 27, no. 7, pp. 645-653, 2014.

[13] F. J. Xie, Y. P. Zhang, Q. Q. Zheng et al., "Helicobacter pylori infection and esophageal cancer risk: an updated meta-analysis," World Journal of Gastroenterology, vol. 19, no. 36, pp. 6098-6107, 2013.

[14] F. Islami and F. Kamangar, "Helicobacter pylori and esophageal cancer risk: a meta-analysis," Cancer Prevention Research, vol. 1, no. 5, pp. 329-338, 2008.

[15] T. Rokkas, D. Pistiolas, P. Sechopoulos, I. Robotis, and G. Margantinis, "Relationship between Helicobacter pylori infection and esophageal neoplasia: a meta-analysis," Clinical Gastroenterology and Hepatology, vol. 5, no. 12, pp. 14131417.e2, 2007.

[16] D. F. Stroup, J. A. Berlin, S. C. Morton et al., "Meta-analysis of observational studies in epidemiology: a proposal for reporting-Meta-analysis Of Observational Studies in Epidemiology (MOOSE) group," JAMA, vol. 283, no. 15, pp. 2008-2012, 2000.

[17] N. J. Talley, A. R. Zinsmeister, A. Weaver et al., "Gastric adenocarcinoma and Helicobacter pylori infection," Journal of the National Cancer Institute, vol. 83, no. 23, pp. 1734-1739, 1991.

[18] S. Öberg, J. H. Peters, J. J. Nigro et al., "Helicobacter pylori is not associated with the manifestations of gastroesophageal 
reflux disease," Archives of Surgery, vol. 134, no. 7, pp. 722726, 1999.

[19] A. P. Weston, A. S. Badr, M. Topalovski, R. Cherian, A. Dixon, and R. S. Hassanein, "Prospective evaluation of the prevalence of gastric Helicobacter pylori infection in patients with GERD, Barrett's esophagus, Barrett's dysplasia, and Barrett's adenocarcinoma," American Journal of Gastroenterology, vol. 95, no. 2, pp. 387-394, 2000.

[20] E. M. El-Omar, C. S. Rabkin, M. D. Gammon et al., "Increased risk of noncardia gastric cancer associated with proinflammatory cytokine gene polymorphisms," Gastroenterology, vol. 124, no. 5, pp. 1193-1201, 2003.

[21] A. Stang, "Critical evaluation of the Newcastle-Ottawa scale for the assessment of the quality of nonrandomized studies in meta-analyses," European Journal of Epidemiology, vol. 25, no. 9, pp. 603-605, 2010.

[22] J. A. Sterne, M. A. Hernan, B. C. Reeves et al., "ROBINS-I: a tool for assessing risk of bias in non-randomised studies of interventions," BMJ, vol. 355, article i4919, 2016.

[23] J. J. Deeks, J. P. Higgins, and D. G. Altman, “Analysing data and undertaking meta-analyses," in Cochrane Handbook for Systematic Reviews of Interventions, J. P. Higgins and S. Green, Eds., pp. 243-296, Wiley, Hoboken, NJ, USA, 2008.

[24] J. IntHout, J. P. Ioannidis, and G. F. Borm, "The HartungKnapp-Sidik-Jonkman method for random effects metaanalysis is straightforward and considerably outperforms the standard DerSimonian-Laird method," BMC Medical Research Methodology, vol. 14, no. 1, p. 25, 2014.

[25] R. DerSimonian and N. Laird, "Meta-analysis in clinical trials," Controlled Clinical Trials, vol. 7, no. 3, pp. 177-188, 1986.

[26] I. J. Vohlonen, M. Hakama, M. Härkönen et al., “Oesophageal cancer incidence in 20-year follow-up in a population-based sample of 12000 middle-age men with or without Helicobacter pylori infection in Finland," Gut, vol. 67, no. 6, pp. 12011202, 2018.

[27] C. H. Tseng, "Metformin and esophageal cancer risk in Taiwanese patients with type 2 diabetes mellitus," Oncotarget, vol. 8, no. 12, pp. 18802-18810, 2017.

[28] A. Sonnenberg, K. O. Turner, S. J. Spechler, and R. M. Genta, "The influence of Helicobacter pylori on the ethnic distribution of Barrett's metaplasia," Alimentary Pharmacology and Therapeutics, vol. 45, no. 2, pp. 283-290, 2017.

[29] O. B. Poyrazoglu, A. C. Dulger, and B. S. Gultepe, "Helicobacter pylory infection in patients with esophageal squamous cell carcinoma," Clinics, vol. 72, no. 3, pp. 150-153, 2017.

[30] S. Obayo, C. Muzoora, P. Ocama, M. M. Cooney, T. Wilson, and C. S. Probert, "Upper gastrointestinal diseases in patients for endoscopy in South-Western Uganda," African Health Sciences, vol. 15, no. 3, pp. 959-966, 2015.

[31] L. Xue, L. Xing, J. Wang et al., "Serum pepsinogens and Helicobacter pylori are not associated with esophageal squamous cell carcinoma in a high-risk area in China," Tumori Journal, vol. 99, no. 2, pp. 134-138, 2013.

[32] G. Murphy, F. Kamangar, D. Albanes et al., "Serum ghrelin is inversely associated with risk of subsequent oesophageal squamous cell carcinoma," Gut, vol. 61, no. 11, pp. 1533-1537, 2012.

[33] M. Venerito, S. Kohrs, T. Wex et al., "Helicobacter pylori infection and fundic gastric atrophy are not associated with esophageal squamous cell carcinoma: a case-control study," European Journal of Gastroenterology \& Hepatology, vol. 23, no. 10 , pp. $859-864,2011$.
[34] M. Khoshbaten, A. Zadimani, M. R. Bonyadi, M. Mohammadzadeh, L. Gachkar, and M. A. Pourhoseingholi, "Helicobacter pylori infection reduces the risk of esophageal squamous cell carcinoma: a case-control study in Iran," Asian Pacific Journal of Cancer Prevention, vol. 12, no. 1, pp. 149151, 2011.

[35] D. C. Whiteman, P. Parmar, P. Fahey et al., "Association of Helicobacter pylori infection with reduced risk for esophageal cancer is independent of environmental and genetic modifiers," Gastroenterology, vol. 139, no. 1, pp. 73-83, 2010.

[36] M. B. Cook, S. M. Dawsey, L. Diaw et al., "Serum pepsinogens and Helicobacter pylori in relation to the risk of esophageal squamous cell carcinoma in the alpha-tocopherol, betacarotene cancer prevention study," Cancer Epidemiology Biomarkers \& Prevention, vol. 19, no. 8, pp. 1966-1975, 2010.

[37] I. C. Wu, D. C. Wu, F. J. Yu et al., "Association between Helicobacter pylori seropositivity and digestive tract cancers," World Journal of Gastroenterology, vol. 15, no. 43, pp. 5465-5471, 2009.

[38] H. M. Hu, C. H. Kuo, C. H. Lee et al., "Polymorphism in COX2 modifies the inverse association between Helicobacter pylori seropositivity and esophageal squamous cell carcinoma risk in Taiwan: a case control study," BMC Gastroenterology, vol. 9, no. 1, p. 37, 2009.

[39] H. E. Löfdahl, Y. Lu, and J. Lagergren, "Sex-specific risk factor profile in oesophageal adenocarcinoma," British Journal of Cancer, vol. 99, no. 9, pp. 1506-1510, 2008.

[40] M. Früh, W. Zhou, R. Zhai et al., "Polymorphisms of inflammatory and metalloproteinase genes, Helicobacter pylori infection and the risk of oesophageal adenocarcinoma," British Journal of Cancer, vol. 98, no. 4, pp. 689-692, 2008.

[41] M. H. Derakhshan, R. Malekzadeh, H. Watabe et al., "Combination of gastric atrophy, reflux symptoms and histological subtype indicates two distinct aetiologies of gastric cardia cancer," Gut, vol. 57, no. 3, pp. 298-305, 2008.

[42] L. A. Anderson, S. J. Murphy, B. T. Johnston et al., "Relationship between ylori infection and gastric atrophy and the stages of the oesophageal inflammation, metaplasia, adenocarcinoma sequence: results from the FINBAR case-control study," Gut, vol. 57, no. 6, pp. 734-739, 2008.

[43] J. H. Simán, L. Engstrand, G. Berglund, A. Forsgren, and C. H. Florén, "Helicobacter pylori and CagA seropositivity and its association with gastric and oesophageal carcinoma," Scandinavian Journal of Gastroenterology, vol. 42, no. 8, pp. 933940, 2007.

[44] F. Kamangar, Y. L. Qiao, M. J. Blaser et al., "Helicobacter pylori and oesophageal and gastric cancers in a prospective study in China," British Journal of Cancer, vol. 96, no. 1, pp. 172-176, 2007.

[45] K. Iijima, T. Koike, Y. Abe et al., "Extensive gastric atrophy: an increased risk factor for superficial esophageal squamous cell carcinoma in Japan," American Journal of Gastroenterology, vol. 102, no. 8, pp. 1603-1609, 2007.

[46] S. Anandasabapathy, J. Jhamb, M. Davila, C. Wei, J. Morris, and R. Bresalier, "Clinical and endoscopic factors predict higher pathologic grades of Barrett dysplasia," Cancer, vol. 109, no. 4, pp. 668-674, 2007.

[47] D. C. Wu, I. C. Wu, J. M. Lee et al., "Helicobacter pylori infection: a protective factor for esophageal squamous cell carcinoma in a Taiwanese population," The American Journal of Gastroenterology, vol. 100, no. 3, pp. 588-593, 2005. 
[48] C. De Martel, A. E. Llosa, S. M. Farr et al., "Helicobacter pylori infection and the risk of development of esophageal adenocarcinoma," The Journal of Infectious Diseases, vol. 191, no. 5, pp. 761-767, 2005.

[49] W. Ye, M. Held, J. Lagergren et al., "Helicobacter pylori infection and gastric atrophy: risk of adenocarcinoma and squamous-cell carcinoma of the esophagus and adenocarcinoma of the gastric cardia," Journal of the National Cancer Institute, vol. 96, no. 5, pp. 388-396, 2004.

[50] A. H. Wu, J. E. Crabtree, L. Bernstein et al., "Role of Helicobacter pylori CagA+ strains and risk of adenocarcinoma of the stomach and esophagus," International Journal of Cancer, vol. 103, no. 6, pp. 815-821, 2003.

[51] K. X. Wang, X. F. Wang, J. L. Peng, Y. B. Cui, J. Wang, and C. P. Li, "Detection of serum anti-Helicobacter pylori immunoglobulin $G$ in patients with different digestive malignant tumors," World Journal of Gastroenterology, vol. 9, no. 11, pp. 2501-2504, 2003.

[52] J. Henrik Simán, A. Forsgren, G. Berglund, and C.-H. Floren, "Helicobacter pylori infection is associated with a decreased risk of developing oesophageal neoplasms," Helicobacter, vol. 6 , no. 4 , pp. 310-316, 2001.

[53] M. Vieth, B. Masoud, A. Meining, and M. Stolte, "Helicobacter pylori infection: protection against Barrett's mucosa and neoplasia?," Digestion, vol. 62, no. 4, pp. 225-231, 2000.

[54] P. M. Peek Jr., M. F. Vaezi, G. W. Falk et al., "Role of Helicobacter pylori cag $A^{+}$strains and specific host immune responses on the development of premalignant and malignant lesions in the gastric cardia," International Journal of Cancer, vol. 82, no. 4, pp. 520-524, 1999.

[55] C. E. Grimley, R. L. Holder, D. E. Loft, A. Morns, and C. U. Nwokolo, "Helicobacter pylori-associated antibodies in patients with duodenal ulcer, gastric and oesophageal adenocarcinoma," European Journal of Gastroenterology and Hepatology, vol. 11, no. 5, pp. 503-510, 1999.

[56] W. H. Chow, M. J. Blaser, W. J. Blot et al., “An inverse relation between cagA+ strains of Helicobacter pylori infection and risk of esophageal and gastric cardia adenocarcinoma," Cancer Research, vol. 58, no. 4, pp. 588-590, 1998.

[57] K. Iijima, T. Koike, Y. Abe et al., "Gastric hyposecretion in esophageal squamous-cell carcinomas," Digestive Diseases and Sciences, vol. 55, no. 5, pp. 1349-1355, 2010.

[58] G. M. Houben and R. W. Stockbrügger, "Bacteria in the aetiopathogenesis of gastric cancer: a review," Scandinavian Journal of Gastroenterology, vol. 30, pp. 13-18, 1995.

[59] J. Kountouras, D. Chatzopoulos, and C. Zavos, "Eradication of Helicobacter pylori might halt the progress to oesophageal adenocarcinoma in patients with gastro-oesophageal reflux disease and Barrett's oesophagus," Medical Hypotheses, vol. 68, no. 5, pp. 1174-1175, 2007.

[60] A. D. Jones, K. D. Bacon, B. A. Jobe, B. C. Sheppard, C. W. Deveney, and M. J. Rutten, "Helicobacter pylori induces apoptosis in Barrett's-derived esophageal adenocarcinoma cells," Journal of Gastrointestinal Surgery, vol. 7, no. 1, pp. 68-76, 2003.

[61] H. G. Coleman, S. H. Xie, and J. Lagergren, "The epidemiology of esophageal adenocarcinoma," Gastroenterology, vol. 154, no. 2, pp. 390-405, 2018.

[62] D. Y. Graham, "Editorial: Helicobacter pylori is not and never was "protective" against anything, including GERD," Digestive Diseases and Sciences, vol. 48, no. 4, pp. 629-630, 2003.
[63] A. Kokkola, T. U. Kosunen, P. Puolakkainen et al., "Spontaneous disappearance of Helicobacter pylori antibodies in patients with advanced atrophic corpus gastritis," APMIS, vol. 111, no. 6, pp. 619-624, 2003.

[64] A. M. Ekström, M. Held, L.-E. Hansson, L. Engstrand, and O. Nyrén, "Helicobacter pylori in gastric cancer established by CagA immunoblot as a marker of past infection," Gastroenterology, vol. 121, no. 4, pp. 784-791, 2001.

[65] X. Zhuo, Y. Zhang, Y. Wang, W. Zhuo, Y. Zhu, and X. Zhang, "Helicobacter pylori infection and oesophageal cancer risk: association studies via evidence-based meta-analyses," Clinical Oncology, vol. 20, no. 10, pp. 757-762, 2008.

[66] A. P. Thrift, "The epidemic of oesophageal carcinoma: where are we now?," Cancer Epidemiology, vol. 41, pp. 88-95, 2016.

[67] S. H. Xie and J. Lagergren, "The male predominance in esophageal adenocarcinoma," Clinical Gastroenterology and Hepatology, vol. 14, no. 3, pp. 338-347.e1, 2016.

[68] S. Take, M. Mizuno, K. Ishiki et al., "Low incidence of esophageal adenocarcinoma after eradication of Helicobacter pylori in Japan," Clinical Gastroenterology and Hepatology, vol. 16, no. 12, pp. 1995-1996, 2018.

[69] Y. Y. Lee, S. Mahendra Raj, and D. Y. Graham, "Helicobacter pylori infection - a boon or a bane: lessons from studies in a low-prevalence population," Helicobacter, vol. 18, no. 5, pp. 338-346, 2013.

[70] W. E. Ek, D. M. Levine, M. D'Amato et al., "Germline genetic contributions to risk for esophageal adenocarcinoma, Barrett's esophagus, and gastroesophageal reflux," Journal of the National Cancer Institute, vol. 105, no. 22, pp. 1711-1718, 2013. 


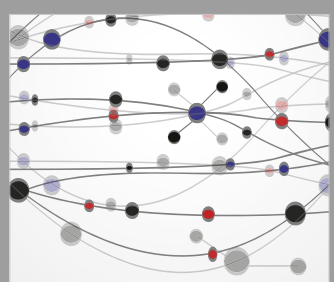

The Scientific World Journal
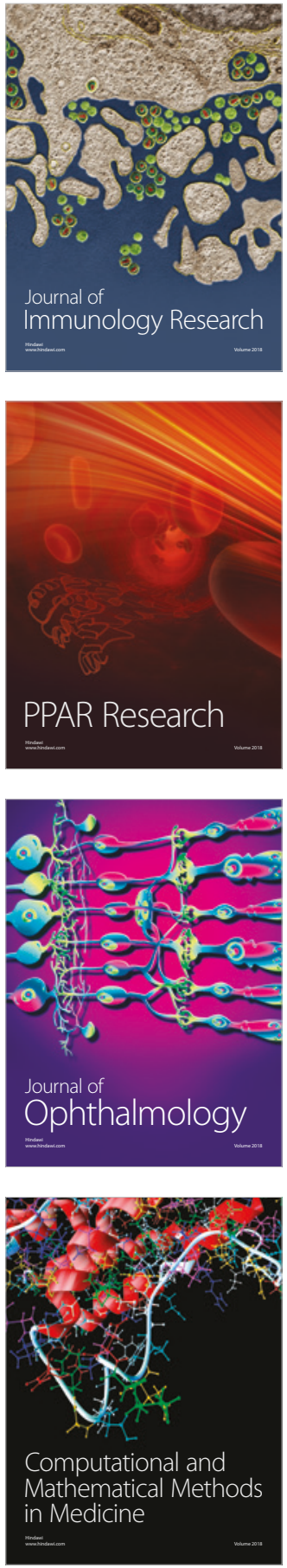

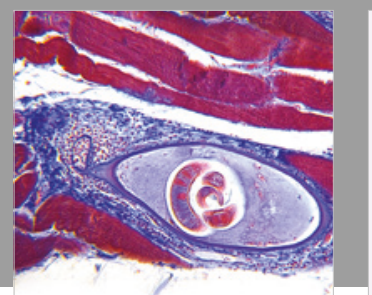

Gastroenterology Research and Practice

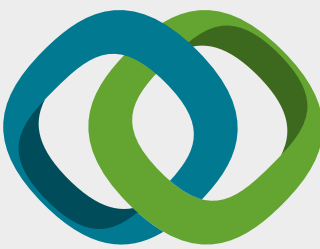

\section{Hindawi}

Submit your manuscripts at

www.hindawi.com
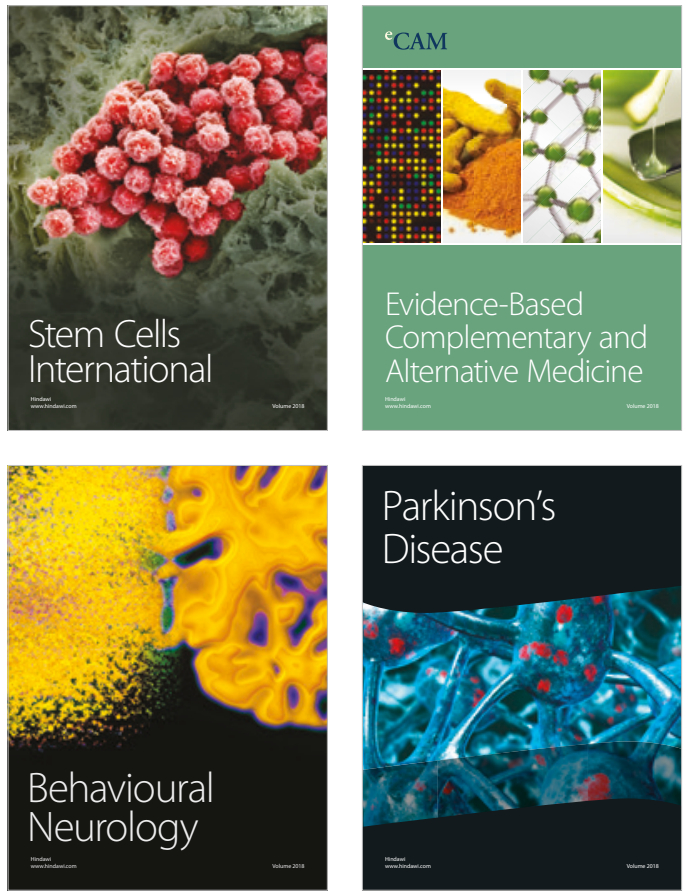

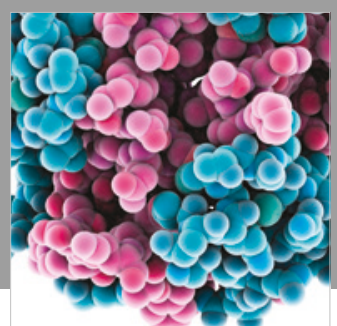

ournal of

Diabetes Research

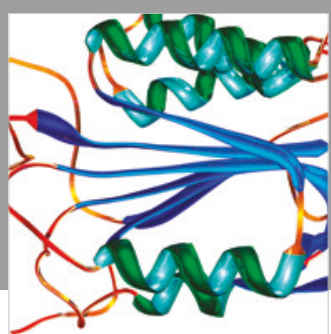

Disease Markers
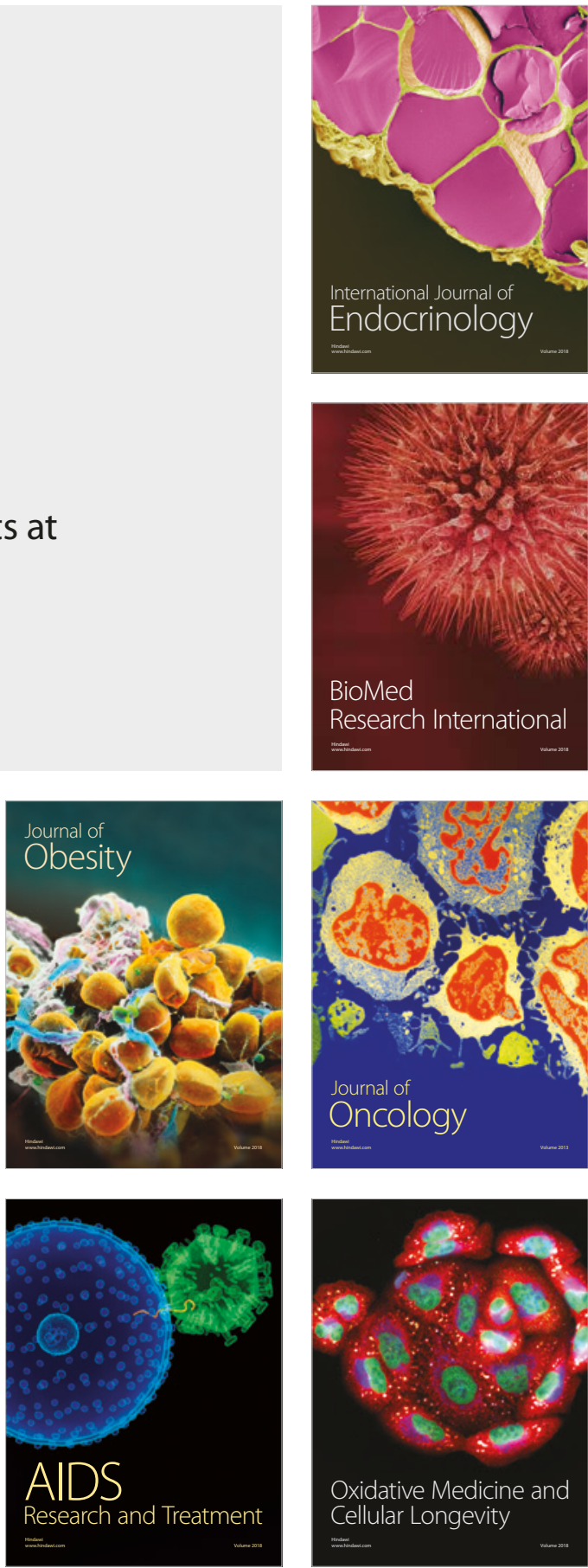